This is a self-archived version of an original article. This version may differ from the original in pagination and typographic details.

Author(s): Bergeret, F. Sebastian; Silaev, Mikhail; Virtanen, Pauli; Heikkilä, Tero

Title: Colloquium : Nonequilibrium effects in superconductors with a spin-splitting field

Year: 2018

Version: Published version

Copyright: @ 2018 American Physical Society

Rights: In Copyright

Rights url: http://rightsstatements.org/page/lnC/1.0/?language=en

Please cite the original version:

Bergeret, F. S., Silaev, M., Virtanen, P., \& Heikkilä, T. (2018). Colloquium : Nonequilibrium effects in superconductors with a spin-splitting field. Reviews of Modern Physics, 90(4), Article 041001. https://doi.org/10.1103/RevModPhys.90.041001 


\title{
Colloquium: Nonequilibrium effects in superconductors with a spin-splitting field
}

\author{
F. Sebastian Bergeret ${ }^{*}$ \\ Centro de Fisica de Materiales (CFM-MPC), Centro Mixto CSIC-UPV/EHU, \\ Manuel de Lardizabal 4, E-20018 San Sebastian, Spain \\ and Donostia International Physics Center (DIPC), \\ Manuel de Lardizabal 5, E-20018 San Sebastian, Spain \\ Mikhail Silaev \\ University of Jyvaskyla, Department of Physics and Nanoscience Center, \\ P.O. Box 35 (YFL), Fl-40014 University of Jyväskylä, Finland
}

Pauli Virtanen

NEST, Istituto Nanoscienze-CNR and Scuola Normale Superiore, I-56127 Pisa, Italy

Tero T. Heikkilä

University of Jyvaskyla, Department of Physics and Nanoscience Center, P.O. Box 35 (YFL), Fl-40014 University of Jyväskylä, Finland

(published 11 October 2018)

\begin{abstract}
This Colloquium discusses the recent progress in understanding the properties of spin-split superconductors under nonequilibrium conditions. Recent experiments and theories demonstrate a rich variety of transport phenomena occurring in devices based on such materials that suggest direct applications in thermoelectricity, low-dissipative spintronics, radiation detection, and sensing. This text discusses different experimental situations and presents a theoretical framework based on quantum kinetic equations. This framework provides an accurate description of the nonequilibrium distribution of charge, spin, and energy, which are the relevant nonequilibrium modes, in different hybrid structures. This Colloquium also reviews experiments on spin-split superconductors and shows how transport measurements reveal the properties of the nonequilibrium modes and their mutual coupling. In particular, the emphasis of the text is on spin injection and diffusion and very large thermoelectric effects in spin-split superconductors.
\end{abstract}

DOI: 10.1103/RevModPhys.90.041001

\section{CONTENTS}

I. Introduction

II. Superconductor with an Exchange Field

A. Overview of the quasiclassical theory of diffusive superconductors

III. Nonequilibrium Modes in Spin-split Superconductors

A. Description of nonequilibrium modes in superconductors with spin splitting

B. Accumulations in terms of the nonequilibrium modes 9

IV. Spin Injection and Diffusion in Superconductors

A. Detection of spin and charge imbalance: Nonlocal transport measurements

B. Nonlocal conductance measurements in spin-split superconductors

C. Spin Hanle effect

D. Spin imbalance by ac excitation

V. Thermoelectric Effects

\footnotetext{
*sebastian_bergeret@ehu.eus

†Tero.T.Heikkila@jyu.fi
}

A. Charge and heat currents at a spin-polarized interface to a spin-split superconductor

B. Linear response: Heat engine based on a superconductor/ferromagnet structure $\quad 14$

C. Spin Seebeck effect 16

D. Thermophase in a S(FI)S contact 16

VI. Summary and Outlook 17

Acknowledgments 18

$\begin{array}{ll}\text { References } & 18\end{array}$

\section{INTRODUCTION}

Ferromagnetism and spin-singlet superconductivity are antagonist orders and hardly coexist in bulk materials. However, hybrid nanostructures allow for the possibility of combining the two phenomena via mutual proximity effects. The combination leads to the emergence of novel features not present in either system alone. We can make a distinction among those characteristics affecting the spectral properties of the materials, showing up when the probed systems are in equilibrium, and those related to nonequilibrium phenomena. 
The emphasis of our text is in the latter phenomena, especially related to steady-state currents or voltages applied across the structures.

Both superconductors and ferromagnets are examples of electron systems with spontaneously broken symmetries and thereby characterized by order parameters. The order parameter for a conventional spin-singlet superconductor is the amplitude of Cooper pairing between electrons in states with opposite spins and momenta (Bardeen, Cooper, and Schrieffer, 1957). The presence of this complex pairing amplitude $F$ leads to two characteristic features of conventional superconductivity (Tinkham, 1996; de Gennes, 1999): An equilibrium supercurrent that is proportional to the gradient of the phase of $F$ and that can be excited without voltage, and to the quasiparticle spectrum exhibiting an energy gap proportional to the absolute value of $F$. The resulting density of states (DOS), [Eq. (1) for $h_{\mathrm{eff}}=0$ ] is strongly energy dependent and results in a nonlinear nonequilibrium response of superconductors.

The main defining features of ferromagnets are the broken spin-rotation symmetry into the direction of magnetization and the associated exchange energy $h$ that splits the spin-up and spin-down spectra. This also leads to a strong spin dependence (spin polarization) of the observables related to ferromagnets.

There are two mechanisms that prevent most of the ferromagnetic materials from becoming superconducting. One of them is the orbital effect due to the intrinsic magnetic field in ferromagnets. When this field exceeds a certain critical value, superconductivity is suppressed
(Ginzburg, 1957). The second mechanism is the paramagnetic effect (Chandrasekhar, 1962; Clogston, 1962; Saint-James, Sarma, and Thomas, 1969). This is due to the intrinsic exchange field of the ferromagnet that shows up as a splitting of the energy levels of spin-up and spin-down electrons and hence prevents the formation of Cooper pairs. We focus here on the regime where this spin-splitting field is present, but not yet too large to kill superconductivity.

In superconductors the spin-splitting field can be generated either due to the Zeeman effect in magnetic field or as a result of the exchange interaction between the electrons forming Cooper pairs and those which determine the magnetic order. Such fields can lead to drastic modifications of the ground state of a spin-singlet superconductor. The best-known example is the formation of the spatially inhomogeneous superconducting state predicted by Fulde and Ferrell (1964) and Larkin and Ovchinnikov (1965) and dubbed as FFLO. Although extensively studied in the literature, the FFLO phase takes place only in a narrow parameter window and therefore its experimental realization is challenging.

Other more robust phenomena related to the spin-splitting fields in superconductors have their origin in the quasiparticle spectrum modification. In the central panel of Fig. 1 we show the resulting spin-split density of states. This was first explored experimentally by Meservey, Tedrow, and Fulde (1970) and Meservey, Tedrow, and Bruno (1975) through the spin-valve effect in the superconductor/ferromagnet $(\mathrm{Al} / \mathrm{Ni})$ tunnel junctions [Fig. 1(a)]. In these experiments the magnetic field was applied in the plane of a thin superconducting film, such that the paramagnetic effect dominates. The spin-split (a) Spin-resolved tunneling

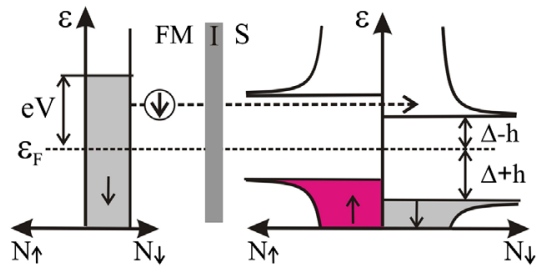

(c) Spin and charge injection

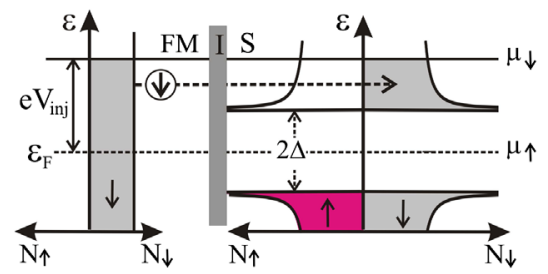

(b) Spin Seebeck effect

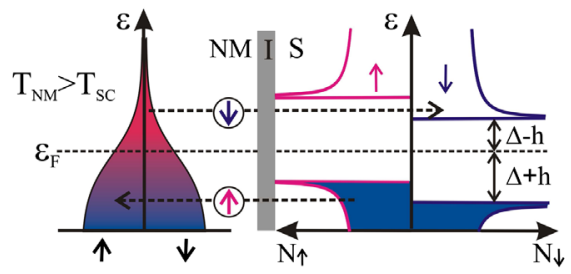

(d) Thermoelectric effect

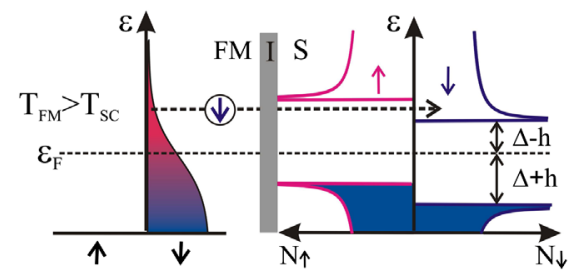

FIG. 1. Central panel: Quasiparticle spectrum and density of states in a superconductor with spin splitting, $N_{0}$ is the normal metal DOS. (a)-(d) Schematic of various nonequilibrium phenomena occurring at normal metal/insulator/superconductor (NM/I/S) and ferromagnetic metal/insulator/superconductor (FM/I/S) interfaces discussed in this review. For clarity we show the limit of half-metallic FM with $N_{\uparrow}=0$. (a) Spin-resolved tunneling from a ferromagnetic metal to a spin-split superconductor that leads to the spin-valve effect, i.e., the charge current in the parallel magnetic configuration is different from that in the antiparallel one. (c) Creation of spin and charge accumulation in the voltage-biased FM/S junction. (b), (d) Schematic of thermally excited currents in NM/S and FM/S junctions with a spin-split superconductor. (b) Spin Seebeck effect in NM/S junction: A pure spin current is generated by the temperature bias between a spin-split superconductor at temperature $T_{\mathrm{SC}}$ and a normal metal at temperature $T_{\mathrm{NM}}>T_{\mathrm{SC}}$. (d) Thermoelectric effect in a FM/I/S junction: Here the spin current is partially converted to the charge current due to the spin-dependent density of states in the ferromagnet. 
DOS was utilized to determine the spin polarization of an adjacent ferromagnet (Tedrow and Meservey, 1971, 1973; Paraskevopoulos, Meservey, and Tedrow, 1977; Meservey, Paraskevopoulos, and Tedrow, 1980; Meservey and Tedrow, 1994). The basis of this spin-valve effect is the spin-resolved tunneling into the superconductor with spin splitting, shown in Fig. 1(a). This schematic illustrates how by properly tuning the voltage across the junction leads to a situation where only one of the spin species participates in electronic transport. That results in peculiar asymmetric differential conductance curves $d I / d V(V) \neq d I / d V(-V)$ observed in experiments and revealing the spin polarization. This idea was used more recently to probe the spatially resolved spin polarization of different magnetic materials by means of scanning tunneling microscopy with spin-split superconducting tips (Eltschka et al., 2014, 2015). Similar effects can also arise in thin superconducting films by the magnetic proximity effect from an adjacent ferromagnetic material (Tedrow, Tkaczyk, and Kumar, 1986). In such a case, the spin splitting of the density of states can be observed for small magnetic fields or even at zero field, as discussed in Sec. II.

The combination of spin-splitting fields with strong spinorbit interaction in superconducting nanowires has also raised considerable interest as a platform for realizing topological phases and Majorana fermions, with possible applications in topological quantum computation (Aasen et al., 2016). Although these effects are beyond the focus of this review, the physics discussed next may help in understanding transport properties of the devices studied in that context.

Because of the different nature of their broken symmetry, combining superconductors $(\mathrm{S})$ and ferromagnets $(\mathrm{FM})$ in hybrid structures leads to a multitude of effects where magnetism affects superconductivity and vice versa. Some of these effects show up already in equilibrium properties, especially studied in the context of proximity effects in superconducting/metallic ferromagnet hybrids and reviewed, for example, by Buzdin (2005) and Bergeret, Volkov, and Efetov (2005). The latter usually focus on the unusual behavior of Cooper pairs leaking from a superconductor into a metallic ferromagnet generating, for example, oscillating pair wave functions analogous to the FFLO state (Buzdin, Bulaevskii, and Panyukov, 1982; Demler, Arnold, and Beasley, 1997) and long-range spin-triplet correlations (Bergeret, Volkov, and Efetov, 2001b) induced by the coupling between the intrinsic exchange field of the ferromagnet and the leaked superconducting condensate (Bergeret, Volkov, and Efetov, 2001b). These effects manifest themselves in measurable equilibrium effects, such as the density of states and critical-temperature oscillations in S/FM bilayers (Jiang et al., 1996; Kontos et al., 2001), triplet spin-valve effects in the critical temperature of FM/S/FM structures (Singh et al., 2015), and unusual Josephson effects in SC/FM/SC junctions (Ryazanov et al., 2001; Singh et al., 2016). Inversely, a magnetic proximity effect can arise when the triplet pairs, created in the FM region, leak back into the superconductor in a FM/S metallic bilayer, generating a nonvanishing magnetic moment in the SC within a coherence length $\xi_{s}$ from the SM/FM interface (Bergeret, Volkov, and Efetov, 2004).

In contrast to these equilibrium proximity effects, here we focus on nonequilibrium properties of a superconducting material with a built-in spin-splitting field. The interest in studying such systems was intensified recently due to the technological advances which allow for a controllable generation of spin splitting in thin superconducting films either by applying an external in-plane magnetic field (Hübler et al., 2012; Quay et al., 2013) or by an adjacent ferromagnetic insulator. Structures with insulating FMs avoid the proximity effect suppressing superconductivity. Such nonequilibrium properties are studied by applying currents or voltages across the structures. The focus of our Colloquium is on steady-state nonequilibrium effects with time-independent driving fields, but we also mention works studying alternating current (ac) responses.

Often the nonequilibrium effects can survive to much higher distances than $\xi_{s}$, as their decay scales are determined via the various inelastic and spin-flip scattering lengths. Moreover, they can be studied at a weak tunneling contact to ferromagnets, making the analysis in some cases more straightforward than in proximity experiments. Nonequilibrium properties are related to the deviation of the electron distribution function from its equilibrium form, which leads to a nonequilibrium distribution (imbalance) of charge, energy, or spin degrees of freedom. We refer to these different types of deviations from equilibrium as nonequilibrium modes. ${ }^{1}$ Specifically, we explore the coupling between these modes in superconductors with a spin-splitting field and discuss unusually strong thermoelectric response and long-range spin signals.

The ability to characterize the spin-polarized Fermi surface of metallic magnets with the help of spin-split superconductors has a direct connection with spintronics, and, in particular, with the search for spin valves with larger efficiencies than in the structures exhibiting large magnetoresistance (Baibich et al., 1988; Binasch et al., 1989; Moodera et al., 1995). Indeed, a superconductor with a spin-splitting field also has an intrinsic energy-dependent spin polarization around the Fermi level. This allows for studying different spintronic effects in the setting of a controllable nonlinearity arising from the superconducting gap. Some of these effects are schematically shown in Fig. 1. This Colloquium explains those phenomena in detail.

In normal metals and superconductors a spin accumulation, or spin imbalance, can be created by injection of a charge current from a ferromagnetic electrode (Johnson and Silsbee, 1985; van Son, van Kempen, and Wyder, 1987; Johnson, 1994; Jedema, Filip, and van Wees, 2001; Gu et al., 2002; Takahashi and Maekawa, 2003; Shin, Lee, and Lee, 2005; Poli et al., 2008). This state is characterized by the excess population in one of the spin subbands, determined by the balance between spin injection and relaxation or spin diffusion rates. In normal metals the nonequilibrium spin imbalance decays due to spin-flip scattering at typical distances of several hundred of nanometers. In the superconducting state,

\footnotetext{
${ }^{1}$ The term "mode" here refers to the changes of the electron distribution function with respect to its equilibrium form. It should be distinguished from collective modes such as the Carlson and Goldman (1973) or the amplitude mode (Higgs, 1964) that affect the response of superconductors at temperatures close to the critical temperature or at high frequencies.
} 
at low temperatures $k_{B} T \ll \Delta$ the injection of any amount of carriers just above the energy gap shifts the chemical potential of quasiparticles rather strongly due to the large amount of quasiparticles at the gap edge [Fig. 1(c)]. This leads to a strong spin signal in S/FM junctions (Takahashi and Maekawa, 2003; Poli et al., 2008).

The spin-relaxation length in normal metals depends only weakly on the temperature $T$. In the superconducting state, however, the scattering length is drastically modified with $T$. According to the first theory and experiments on spin injection in superconductors, the spin-relaxation length was found to be reduced compared to the normal state (Morten, Brataas, and Belzig, 2004; Poli et al., 2008). However, subsequent experiments showed, contrary to expectations, an increase of the spin decay length (Hübler et al., 2012; Quay et al., 2013). It is now understood that these findings can be explained only by taking into account the spin-splitting field inside the superconductor (Bobkova and Bobkov, 2015, 2016; Krishtop, Houzet, and Meyer, 2015; Silaev, Virtanen, Bergeret, and Heikkilä, 2015). Because of this field, as shown in Sec. III.B, it is necessary to take into account four types of nonequilibrium modes describing spin, charge, energy, and spin-energy imbalances. These modes provide the natural generalization of the charge and energy imbalances introduced by Schmid and Schön (1975). In Sec. IV we show how the spin-splitting field couples pairwise to these modes: charge to spin energy and spin to energy. Such a coupling leads to striking effects. For example, the coupling between the spin and energy modes leads to the long-range spin accumulation observed in the experiments by Hübler et al. (2012) and Quay et al. (2013). As we show in Sec. IV this long-range effect is related to the fact that the energy mode can relax only via inelastic processes which at low temperatures are rare.

The coupling between different modes shows up also in tunnel contacts with spin-split superconductors. Because the spin-splitting field shifts the spin-resolved DOS away from the chemical potential of the superconductor, the system exhibits a strong spin-dependent electron-hole asymmetry. The spinaveraged density of states is still electron-hole symmetric and therefore does not violate fundamental symmetries of the quasiclassical superconducting state. This spin-resolved electron-hole asymmetry leads to a large spin Seebeck effect shown schematically in Fig. 1(b) and discussed in Sec. V.C. A temperature difference across a tunneling interface between a normal metal and a spin-split superconductor drives a pure spin current between the electrodes, without transport of charge. If one of the electrodes is small so that the spin injection rate is large or comparable to the rate for spin relaxation, a spin accumulation forms in this electrode.

However, it was noticed in several recent works (Kalenkov, Zaikin, and Kuzmin, 2012; Machon, Eschrig, and Belzig, 2013, 2014; Ozaeta et al., 2014) that in certain situations the relevant observables are not spin averaged, resulting in an effective electron-hole asymmetry showing up also in the charge current. The spin components are weighted differently in a setup consisting of the spin-filter junction connected to the spin-split superconductor (Ozaeta et al., 2014), shown schematically in Fig. 1(d). As a result of this effective electronhole symmetry breaking, the system exhibits a very large thermoelectric effect. This is discussed in Sec. V.
The main body of this Colloquium is organized as follows. In Sec. II we describe spin-split superconductors and give an overview of the quasiclassical theory that can be used for describing both their equilibrium and their nonequilibrium properties. In Sec. III we describe the nonequilibrium modes in superconducting systems driven out of equilibrium in terms of the quasiclassical formalism. Section IV focuses on the spin injection and diffusion in superconducting systems and reviews experiments performed to detect spin and charge imbalance in superconductors with and without spin splitting. In Sec. $\mathrm{V}$ we describe the giant thermoelectric response of a system exhibiting spin-polarized tunneling into a superconductor with a spin-splitting field. Finally, we present our conclusions and an outlook on possible future developments in the field in Sec. VI. A longer version of this Colloquium, along with comprehensive technical detail, can be found in Bergeret et al. (2017).

\section{SUPERCONDUCTOR WITH AN EXCHANGE FIELD}

The main focus of this Colloquium is on superconductors with a spin-split DOS. As discussed in the Introduction such a splitting can originate either by an external magnetic field (Meservey, Tedrow, and Fulde, 1970) or by the exchange field induced by an adjacent ferromagnetic insulator (Tedrow, Tkaczyk, and Kumar, 1986). The split DOS was observed in spectroscopy experiments (Meservey, Tedrow, and Fulde, 1970; Tedrow and Meservey, 1971; Paraskevopoulos, Meservey, and Tedrow, 1977; Meservey, Paraskevopoulos, and Tedrow, 1980; Hao, Moodera, and Meservey, 1990; Xiong et al., 2011).

Formally, the normalized DOS of a spin-split BardeenCooper-Schrieffer (BCS) superconductor is expressed as the sum of the DOS of each spin species $N=N_{\uparrow}+N_{\downarrow}$,

$N=\frac{1}{2} \operatorname{Re} \frac{\varepsilon+h_{\text {eff }}}{\sqrt{\left(\varepsilon+h_{\text {eff }}\right)^{2}-\Delta^{2}}}+\frac{1}{2} \operatorname{Re} \frac{\varepsilon-h_{\text {eff }}}{\sqrt{\left(\varepsilon-h_{\text {eff }}\right)^{2}-\Delta^{2}}}$,

where $\pm h_{\text {eff }}$ is the effective spin-splitting field. Equation (1) is a simplified description because it does not take into account the effect of magnetic impurities or spin-orbit coupling (SOC) (Meservey and Tedrow, 1994) discussed in the next pages. Often inelastic processes are described by $\varepsilon \mapsto \varepsilon+i \Gamma$, where $\Gamma$ is the Dynes et al. (1984) parameter.

In the case when the exchange field is induced by an adjacent ferromagnetic insulator (FI) there is no need of applying an external magnetic field (Tedrow, Tkaczyk, and Kumar, 1986; Hao, Moodera, and Meservey, 1990; Moodera, Santos, and Nagahama, 2007; Senapati, Blamire, and Barber, 2011; Xiong et al., 2011; Wolf, Sürgers et al., 2014). Microscopically, the spin splitting originates from the exchange interaction between conduction electrons and the magnetic moments of the FI localized at the S/FI interface (Tokuyasu, Sauls, and Rainer, 1988; Khusainov, 1996; Izyumov, Proshin, and Khusainov, 2002). The ferromagnetic ordering in the FI is due to a direct exchange coupling between the localized magnetic moments. In usual FIs the direct coupling is strong enough that one can assume that the magnetic configuration of the FI is only weakly affected by 
the superconducting state (Buzdin and Bulaevskii, 1988; Bergeret, Efetov, and Larkin, 2000).

The modification of the DOS is nonlocal and survives over distances away from the FI/S interface of the order of the coherence length $\xi_{s}$ (Tokuyasu, Sauls, and Rainer, 1988; Bergeret, Volkov, and Efetov, 2004). If the thickness $d$ of the S film is much smaller than $\xi_{s}$, the spin splitting can be assumed as homogeneous across the film. Thus the density of states can be approximated by Eq. (1) with an effective exchange field $\mathbf{h}_{\text {eff }} \approx J_{\text {ex }}\left\langle\mathbf{S}_{\mathbf{r}}\right\rangle a / d$ (de Gennes, 1966; Tokuyasu, Sauls, and Rainer, 1988; Khusainov, 1996), where $a$ is the characteristic distance between the localized spins, $J_{\text {ex }}$ is the exchange coupling between conduction electrons and localized moments, and $\left\langle\mathbf{S}_{\mathbf{r}}\right\rangle$ is the average of the latter.

In Fig. 2 we show an example of the measured differential conductance of an $\mathrm{EuS} / \mathrm{Al} / \mathrm{Al}_{2} \mathrm{O}_{3} / \mathrm{Al}$ junction. The $\mathrm{Al}$ layer adjacent to the EuS has a spin-split density of states that shows up as the splitting peaks (bright stripes in the figure) in $d I / d V$. Even at zero applied magnetic field the splitting is nonzero. The magnetization reversal of EuS at $H_{c} \approx-18.5 \mathrm{mT}$ manifests as a discontinuity of the conductance peaks (Strambini et al., 2017). As a first approximation, the DOS in Eq. (1) describes the results in Fig. 2 quite well.

The advantage of using a FI instead of an external magnetic field is that one avoids the depairing effects and all complications caused by the need to apply magnetic fields in superconducting devices. Moreover, because the electrons of the superconductor cannot propagate into the FI, superconducting properties are only modified by the induced spinsplitting field at the S/FI interface, and not by the leakage of Cooper pairs into the FI as would happen in the case of metallic ferromagnets. In addition, FIs can also be used as spin-filter barriers (Moodera, Santos, and Nagahama, 2007),

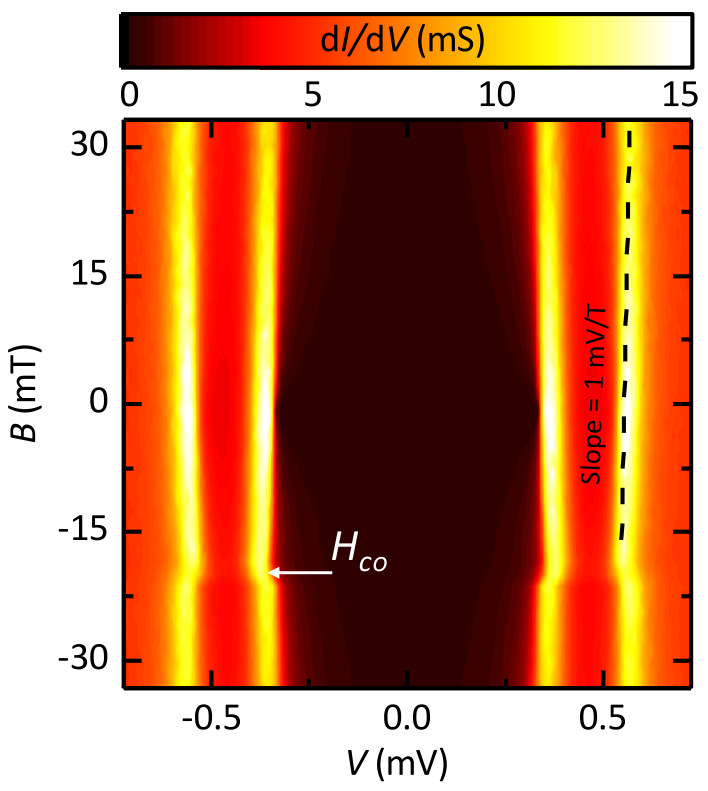

FIG. 2. Measured differential conductance $d I / d V$ of a $\mathrm{EuS} / \mathrm{Al} / \mathrm{Al}_{2} \mathrm{O}_{3} / \mathrm{Al}$ junction as a function of the applied voltage and external magnetic field. $H_{\text {co }}$ denotes the coercive field of the EuS layer when the magnetization switches. Adapted from Strambini et al., 2017. in some cases with a very high spin-filtering efficiency, and therefore they play a crucial role in different applications as discussed next.

In Table I we show a list of FI/S combinations and the reported induced exchange splittings and spin-filter efficiencies (barrier spin polarizations).

The paramagnetic effect, that leads to the spin splitting, is modified by spin relaxation and orbital depairing. In their absence the superconductivity survives the spin-splitting field up to the Chandrasekhar-Clogston limit (Chandrasekhar, 1962; Clogston, 1962) $h=\Delta_{0} / \sqrt{2}$, where $\Delta_{0}$ is the order parameter at zero field and zero temperature. At this field the system experiences a first-order phase transition into the normal state when the order parameter changes abruptly from $\Delta_{0}$ to zero. This picture changes qualitatively due to the presence of magnetic impurities and spin-orbit scattering. Even at $T=0$ and $h=0$ the spin-flip processes induced by magnetic impurities result in the pair breaking effect closing the energy gap (Abrikosov and Gor'kov, 1960a) at $\tau_{\mathrm{sf}} \Delta_{0}=$ $3 / 4$ and suppressing superconductivity completely at a certain critical value of the spin-flip time $\tau_{\mathrm{sf}}$. For values of $\tau_{\mathrm{sf}}$ larger than the critical one the phase transition switches from the first to the second order at (Bruno, Ronald and Schwartz, 1973) $\tau_{\mathrm{sf}} \Delta_{0}=0.461$ and the gapless state appears at a certain value of $h\left(\tau_{\text {sf }}\right)$ [see Fig. 3(a)].

Contrary to the spin-flip processes, the spin-orbit scattering alone does not have any effect on the superconducting state. However, in combination with $h \neq 0$ it tends to smear out the spin-splitted DOS singularities provided the spin-orbit relaxation time $\tau_{\text {so }}$ is not very short [see Fig. 3(b)]. At short relaxation times $\tau_{\text {so }} \ll \Gamma / \Delta^{2}$, where $\Gamma$ is the depairing parameter (Dynes et al., 1984), the effect of spin splitting is eliminated, and the usual BCS density of states is recovered [see Fig. 3(b)]. Therefore in this case the critical spin-splitting field is strongly increased above the Chandrasekhar-Clogston limit (Bruno, Ronald and Schwartz, 1973).

TABLE I. Magnetic properties of different ferromagnetic insulatorsuperconductor junctions used in experiments. The middle column shows the spin-filter efficiency characterized by the polarization $P=\left(G_{\uparrow}-G_{\downarrow}\right) /\left(G_{\uparrow}+G_{\downarrow}\right)$ of the FI barrier (italics) with normalstate conductance $G_{\sigma}$ for spin $\sigma$. The exchange splittings measured in the superconductor (bold) are listed in the right column. Note that $\mu_{B} \cdot 1 \mathrm{~T}=58 \mu \mathrm{eV} \cong 670 \mathrm{mK}$.

\begin{tabular}{lcc}
\hline \hline $\begin{array}{l}\text { Material } \\
\text { combination }\end{array}$ & $\begin{array}{c}\text { Barrier } \\
\text { polarization }\end{array}$ & $\begin{array}{c}\text { Exchange splitting } \\
\text { (applied field) }\end{array}$ \\
\hline$E u O / \mathbf{A l} / \mathrm{AlO}_{3} / \mathrm{Al}^{1}$ & $\begin{array}{c}\text { No spin-filter } \\
\text { barrier }\end{array}$ & $1(0.1 \mathrm{~T})-1.73 \mathrm{~T}(0.4 \mathrm{~T})$ \\
$\mathrm{Au} / \mathrm{EuS} / \mathrm{Al}^{2}$ & 0.8 & $1.6 \mathrm{~T}(0 \mathrm{~T})$ \\
$\mathrm{Al} / \mathrm{EuS} / \mathrm{Al}^{3}$ & $0.6-0.85$ & $1.9-2.6 \mathrm{~T}(0 \mathrm{~T})$ \\
$\mathrm{Ag} / \mathrm{EuSe} / \mathbf{A l}^{4}$ & $>0.97$ & None at zero field \\
$\mathrm{EuSe} / \mathrm{Al} / \mathrm{AlO}_{3} / \mathrm{Ag}^{4}$ & No spin-filter & $4 \mathrm{~T}(0.6 \mathrm{~T})$ \\
& barrier & \\
$\mathbf{N b N} / G d N / \mathbf{N b N}^{5}$ & 0.75 & $1.4 \mathrm{~T}(0 \mathrm{~T})$ \\
$\mathbf{N b N} / G d N / \mathrm{TiN}^{6}$ & 0.97 & \\
\hline \hline
\end{tabular}

${ }^{1}$ Tedrow, Tkaczyk, and Kumar (1986).

${ }^{2}$ Moodera et al. (1988).

${ }^{3}$ Hao, Moodera, and Meservey (1990).

${ }^{4}$ Moodera, Meservey, and Hao (1993).

${ }^{5}$ Senapati, Blamire, and Barber (2011).

${ }^{6} \mathrm{Pal}$ and Blamire (2015). 

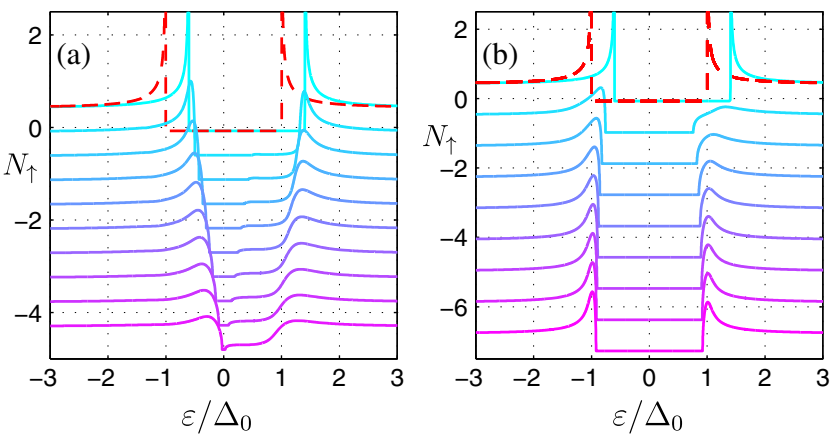

FIG. 3. Calculated density of states of a thin superconducting film at $T \rightarrow 0$. We show the DOS for only one of the spin species $N_{\uparrow}$. Shown by dashed red lines is the DOS in the absence of relaxation $\tau_{\mathrm{sn}}=1 /\left(\tau_{\mathrm{sf}}^{-1}+\tau_{\mathrm{so}}^{-1}\right)=\infty$ and zero exchange field $h=0$ resulting into a gap $\Delta_{0}$. Other curves are plotted for $h=$ $0.4 \Delta_{0}$ and different spin-relaxation rates. (a) Spin-flip relaxation $\beta=\left(\tau_{\mathrm{so}}-\tau_{\mathrm{sf}}\right) /\left(\tau_{\mathrm{so}}+\tau_{\mathrm{sf}}\right)=1$; curves from top to bottom correspond to an increasing $\left(\tau_{\mathrm{sn}} \Delta_{0}\right)^{-1}$, varying equidistantly from 0 by 0.04 steps. (b) Spin-orbit relaxation $\beta=-1$; curves from top to bottom correspond to an increasing $\left(\tau_{\mathrm{sn}} \Delta_{0}\right)^{-1}$, varying equidistantly from 0 by steps of 3.4 . For clarity the curves are shifted along the vertical axis.

Besides broadening of the DOS singularities, the spin-orbit and spin-flip relaxation processes have an important effect on the paramagnetic spin susceptibility of the superconductor as it becomes nonvanishing even in the zero-temperature limit (Yosida, 1958; Abrikosov and Gor'kov, 1960b; Bruno, Ronald and Schwartz, 1973). The static spin susceptibility characterizes the paramagnetic response of the superconductor to an external magnetic field. In a usual normal metal the Zeeman field produces the same magnetization as a spindependent chemical potential shift $\delta \mu$ of the same magnitude when the distribution functions in different spin subbands are given by $f_{\uparrow}(E)=f_{0}(E+\delta \mu)$ and $f_{\downarrow}(E)=f_{0}(E-\delta \mu)$. This is different in superconductors where the paramagnetic susceptibility is determined by both the spin-polarized quasiparticles and the emergent spin-triplet superconducting correlations (Abrikosov and Gor'kov, 1960b, 1962). On the other hand, the nonequilibrium spin modes as systematically described in Sec. III are determined only by the quasiparticle contribution.

In the next sections we review the transport properties of diffusive hybrid structures with spin-split superconductors by contrasting existing theories and experiments. For this sake, in the next section we briefly introduce the quasiclassical Green's function (GF) formalism for superconductors in the presence of spin-dependent fields and spin-polarized interfaces. It is in our opinion the most suitable formalism for the description of diffusive hybrid structures.

\section{A. Overview of the quasiclassical theory of diffusive superconductors}

The quasiclassical Keldysh Green's function technique is a useful and well-established way to describe transport and nonequilibrium properties of good metals, where the relevant physical length scales affecting different observables are long compared to the Fermi wavelength $\lambda_{F}$, and where, in particular, disorder plays a major role. Several reviews explain this technique for various applications (Belzig et al., 1999; Bergeret, Volkov, and Efetov, 2005). Here we outline just the main features relevant for spin-split superconductors. Briefly, the Keldysh GFs $\breve{G}\left(\mathbf{r}, \mathbf{r}^{\prime} ; \mathbf{t}, \mathbf{t}^{\prime}\right)$ are two-point correlation functions which depend on two coordinates and two times. Here the "check" $\breve{G}$ denotes GFs that live in a structure formed by the direct product of Keldysh, spin, and Nambu spaces. The equation of motion for $\breve{G}$ can be written as a kineticlike equation for the Wigner transformed GF $\breve{G}(\mathbf{R}, \mathbf{p})$, where $\mathbf{R}$ is the center of mass coordinate and $\mathbf{p}$ is the momentum after Fourier transformation with respect to the relative component. A significant simplification can be done in the case of metals by noticing that the Green's functions are peaked at the Fermi level. This allows for an integration of the equations over the quasiparticle energy, related to the magnitude of $\mathbf{p}$. This procedure leads to the quasiclassical GFs $\check{g}(\mathbf{R}, \mathbf{n})$, which depend only on the direction of the momentum at the Fermi level and on two times in the case of nonstationary problems, or only on a single energy $\varepsilon$ in the stationary case. These functions obey the Eilenberger (1968) equation. One of the advantages of using the quasiclassical GFs is that, in the normal state, the spectral part is trivial, i.e., the retarded and advanced GFs are energy independent. All transport information of the normal metal is encoded in the quasiclassical Wigner distribution function $\hat{f}(\mathbf{R}, \mathbf{n})$ and quasiclassical equation for it resembles the classical Boltzmann equation (Langenberg and Larkin, 1986).

In contrast, the superconducting case distinguishes itself by a nontrivial spectrum and therefore requires taking into account the full Keldysh structure of the GFs, i.e.,

$$
\check{g}=\left(\begin{array}{cc}
\hat{g}^{R} & \hat{g}^{K} \\
0 & \hat{g}^{A}
\end{array}\right) .
$$

This GF satisfies the normalization condition (Eilenberger, 1968)

$$
\check{g}^{2}=\check{1}
$$

In the diffusive limit the elastic mean free path $l$ due to scattering at nonmagnetic impurities is much smaller than any other length involved in the problem except $\lambda_{F}$. Within this limit the Eilenberger equation can be reduced to a diffusivelike equation in the same way as the Boltzmann equation is simplified in the diffusive limit. This quasiclassical diffusion equation for superconductors is the Usadel (1970) equation (we set $\hbar=k_{B}=1$ )

$$
D \nabla \cdot(\check{g} \nabla \check{g})+\left[i \varepsilon \tau_{3}-i \boldsymbol{h} \cdot \boldsymbol{\sigma} \tau_{3}-\check{\Delta}-\check{\Sigma}, \check{g}\right]=0 .
$$

Here $D$ is the diffusion coefficient, $\breve{g}(\mathbf{r}, \varepsilon)$ is the isotropic (momentum independent) quasiclassical GF, $\boldsymbol{h}$ is the spinsplitting field generated either by an external field or by the magnetic proximity effect in a FI/S junction, and $\check{\Delta}=$ $\Delta e^{i \varphi \tau_{3}} \tau_{1}$ depends on the superconducting order parameter $\Delta$ that has to be determined self-consistently. Here $\tau_{i}$ and $\sigma_{i}$ 
are Pauli spin matrices in Nambu and spin space, respectively. The self-energy $\check{\Sigma}$ in Eq. (4) describes different scattering processes, such as elastic spin-flip or spin-orbit scattering $\check{\Sigma}_{\text {el }}$ and inelastic electron-phonon and electron-electron scattering $\check{\Sigma}_{\text {in }}$.

Equation (4) is central in the description of diffusive superconducting structures. Whereas the spectral properties can be obtained by solving the retarded $(R)$ and advanced $(A)$ components of this equation, nonequilibrium properties are described by the kinetic equation obtained by taking the Keldysh $(K)$ component of Eq. (4). This can be compactly written as

$$
\nabla_{k} j_{k b}^{a}=H^{a b}+R^{a b}+I_{\text {coll }}^{a b},
$$

where we introduce the spectral current tensor $j_{k b}^{a}$,

$$
j_{k b}^{a}=\frac{1}{8} \operatorname{Tr} \tau_{b} \sigma_{a}\left(\check{g} \nabla_{k} \check{g}\right)^{K} .
$$

The different current density components (charge, spin, energy, spin energy) can be obtained from Eq. (6). For example, the charge current density reads

$$
J_{k}=\frac{\sigma_{N}}{2 e} \int_{-\infty}^{\infty} d \varepsilon j_{k 3}^{0} .
$$

Here $\sigma_{N}=e^{2} \nu_{F} D$ and $\nu_{F}$ are the normal-state conductivity and density of states at the Fermi level, respectively. In Eq. (5) the term $H^{a b}=\operatorname{Tr} \tau_{b} \sigma_{a}\left[-i \mathbf{h} \cdot \boldsymbol{\sigma} \tau_{3}, \hat{g}^{K}\right] / 8$ describes the Hanle precession of spin caused by the exchange field, and $R^{a b}=$ $\operatorname{Tr} \tau_{b} \sigma_{a}\left[\hat{\Delta}, \hat{g}^{K}\right] / 8$ the conversion between quasiparticles and the superconducting condensate. Finally $I_{\text {coll }}^{a b}=\operatorname{Tr} \tau_{b} \sigma_{a}[\check{\Sigma}, \check{g}]^{K} / 8$ in Eq. (5) is the collision integral describing the different scattering process with self-energy $\check{\Sigma}$. We next discuss different scattering processes.

Elastic self-energy terms: We consider elastic contributions to $\check{\Sigma}_{\text {el }}$ due to scattering at impurities with spin-orbit coupling (relaxation time $\tau_{\text {so }}$ ) and the spin flips at magnetic impurities $\left(\tau_{\text {sf }}\right)$ (Maki, 1966). Within the Born approximation, they read $\check{\Sigma}_{\text {so }}=\boldsymbol{\sigma} \cdot \check{g} \boldsymbol{\sigma} /\left(8 \tau_{\mathrm{so}}\right), \check{\Sigma}_{\mathrm{sf}}=\boldsymbol{\sigma} \cdot \tau_{3} \check{g} \tau_{3} \boldsymbol{\sigma} /\left(8 \tau_{\mathrm{sf}}\right)$. In the normal state they contribute to the energy-independent total spinrelaxation time $\tau_{\mathrm{sn}}^{-1}=\tau_{\mathrm{so}}^{-1}+\tau_{\mathrm{sf}}^{-1}$. In contrast, in the superconducting case the spin-relaxation time and length acquire energy dependence, which is different for the spin-orbit and spin-flip scattering (Maki, 1966; Morten, Brataas, and Belzig, 2004, 2005). Therefore it is convenient to describe the relative strength of these two scattering mechanisms in terms of the parameter $\beta=\left(\tau_{\text {so }}-\tau_{\text {sf }}\right) /\left(\tau_{\text {so }}+\tau_{\text {sf }}\right)$. In diffusive superconducting thin films one can also describe the depairing effect of an in-plane magnetic field with a self-energy term $\check{\Sigma}_{\text {orb }}=$ $\tau_{3} \check{g} \tau_{3} / \tau_{\text {orb }}$ characterized by the orbital depairing time $\tau_{\text {orb }}$ (de Gennes, 1999; Anthore, Pothier, and Esteve, 2003). This term also contributes to charge imbalance relaxation (Schmid and Schön, 1975; Nielsen et al., 1982).

The parameters $\tau_{\mathrm{sn}}^{-1}$ and $\beta$ are material specific. For example, in $\mathrm{Al}$ films, the reported values from a set of spin injection experiments are $\tau_{\mathrm{sn}} \approx 100$ ps (Jedema et al., 2002; Poli et al., 2008) and $\beta \approx 0.5$ indicating the dominance of spin-flip relaxation over spin-orbit scattering, whereas the reported value of $\tau_{\mathrm{sn}}$ in $\mathrm{Nb}$ is only $0.2 \mathrm{ps}$ and is strongly dominated by spin-orbit scattering (Wakamura et al., 2014). They affect both the spectrum of a bulk superconductor (see Fig. 3) and the spin relaxation as described in Sec. IV.A.

Inelastic self-energies: The relevant inelastic processes entering the self-energy in Eq. (4) are the particle-phonon and particle-particle collisions. These processes do not conserve the energies of colliding quasiparticles, but conserve the total spin.

The coupling between quasiparticles and phonons limits some of the effects discussed in the following sections. Because of the energy dependence of the phonon density of states, this coupling decreases rapidly toward low temperatures, and eventually phonons decouple from electrons, and the main heat relaxation occurs via other processes such as quasiparticle diffusion. Superconductivity modifies the electron-phonon heat conduction (Eliashberg, 1972; Kaplan et al., 1976; Kopnin, 2001), as also the electronic spectrum is energy dependent and is affected by the spin splitting (Grimaldi and Fulde, 1997; Virtanen, Heikkilä, and Bergeret, 2016).

Particle-particle collisions in superconductors and superfluids are discussed by Eliashberg (1972), Serene and Rainer (1983), and Kopnin (2001), although mainly within contact interaction models disregarding screening effects (Narozhny, Aleiner, and Altshuler, 1999; Feigel'man, Larkin, and Skvortsov, 2000; Kamenev and Levchenko, 2009). The collision integrals can have spin structure also in the normal state (Chtchelkatchev and Burmistrov, 2008; Dimitrova and Kravtsov, 2008).

The far-from-equilibrium results discussed in Sec. IV disregard the particle-particle collisions, as the simpler theory already describes effects not very far from the measured ones. On the other hand, Sec. V mostly concentrates on the quasiequilibrium limit, where spin accumulation is also lost due to a strong spin relaxation.

Hybrid interfaces: In subsequent sections we apply the kinetic equation (5) in different situations. For the description of transport in hybrid structures, we need in addition a description of interfaces between different materials in the form of boundary conditions. Such interfaces usually are described by sharp changes of the potential and material parameters over atomic distances and thus cannot be included directly in the quasiclassical equations which describe properties over distances much larger than $\lambda_{F}$. The description of hybrid interfaces requires then the derivation of suitable boundary conditions first done in the quasiclassical approach by Zaitsev (1984).

Boundary conditions for the Usadel equation trace back to the work of Kuprianov and Lukichev (1988). These boundary conditions are applicable for nonmagnetic $N-N, S-S$, and $S-N$ interfaces with low transmissivity (Lambert et al., 1997). Later Nazarov (1999) generalized these boundary conditions for an arbitrary interface transparency.

Tokuyasu, Sauls, and Rainer (1988) derived the boundary condition for an interface between a superconductor and a ferromagnetic insulator and introduced the concept of the spin-mixing angle, which describes the spin-dependent phase shifts acquired by the electrons after being scattered at the FI/S interface. Later Zhao, Löfwander, and Sauls (2004) and Cottet et al. (2009) extended these boundary conditions to magnetic 
metallic structures, such as F-S or S-F-S systems, although with low polarization. Boundary conditions for large polarization and low transmission have been presented by Bergeret, Verso, and Volkov (2012) and Machon, Eschrig, and Belzig (2013). General boundary conditions for arbitrary spin polarization and transmission have been extensively discussed by Eschrig et al. (2015).

Here we mainly deal with tunneling barriers with low transmission between a mesoscopic superconductor and normal and magnetic leads and use the description presented by Bergeret, Verso, and Volkov (2012). In this description, the component of the spectral current density multiplied by $\sigma_{N}$ perpendicular to the interface is continuous across it and given by

$$
\sigma_{N} j_{\perp, b}^{a}=-\frac{1}{8 e R_{\square}} \operatorname{Tr} \tau_{b} \sigma_{a}\left[\hat{\Gamma}_{\breve{g}} \hat{\Gamma}^{\dagger}, \check{g}\right]^{K},
$$

where $R_{\square}$ is the spin-averaged barrier resistance per unit area, and the spin-dependent transmission is characterized by the tunneling matrix $\hat{\Gamma}=t \tau_{3}+u \sigma_{3}$, assuming polarization in the $z$ direction. The normalized transparencies satisfy $t^{2}+u^{2}=1$ and are determined from the interface polarization $|P| \leq 1$ via $2 u t=P$. The Green's function $\check{g}_{2}$ on the right-hand side (rhs) of Eq. (8) corresponds to the opposite side of the junction.

\section{NONEQUILIBRIUM MODES IN SPIN-SPLIT SUPERCONDUCTORS}

The out-of-equilibrium state in superconducting systems is characterized by the presence of nonequilibrium modes associated with the different electronic degrees of freedom. For example, injection of an electric current from a normal electrode into a superconductor generates a charge imbalance mode (Clarke, 1972; Tinkham, 1972; Tinkham and Clarke, 1972; Yagi, 2006; Hübler et al., 2010) that diffuses into the $S$ region. This nonequilibrium mode reflects an imbalance of the quasiparticle population between the electronlike and the holelike spectrum branches. The charge imbalance measurements made in the 1970s were to our knowledge the first to study such nonequilibrium modes in nonlocal multiterminal settings. This technique was later adapted to spintronics to study the nonequilibrium spin accumulation induced by spinpolarized electrodes (Johnson and Silsbee, 1985).

Schematically, nonequilibrium modes can be represented in terms of the electron or hole branches in the spectrum of the superconductor (Tinkham, 1996), as illustrated in Fig. 4. For example, the charge mode can be understood as the imbalance between the electron and hole branches [Fig. 4(b)]. In the absence of spin-dependent fields there is one more nonequilibrium mode: the energy imbalance mode [Fig. 4(e)]. It describes the excess energy stemming from an equal change in the quasiparticle populations of the electronlike and holelike branches. This energy mode affects charge transport properties indirectly via the self-consistency equation for $\Delta$. This mechanism explains, for example, the enhancement of the superconducting transition temperature in the presence of a microwave field (Ivlev, Lisitsyn, and Eliashberg, 1973; Klapwijk, Van den Bergh, and Mooij, 1977).

In this section we generalize the description in terms of nonequilibrium modes to account for superconductors with spin-split density of states. The spin splitting (energy difference $2 h=2 \mu_{B} B$ between the black and red dispersion curves in Fig. 4 for spin-up and spin-down quasiparticles) gives rise to four distinct quasiparticle branches (electron or hole and spin up or down) and the same number of the distribution function components. These four nonequilibrium modes and (a) Equilibrium

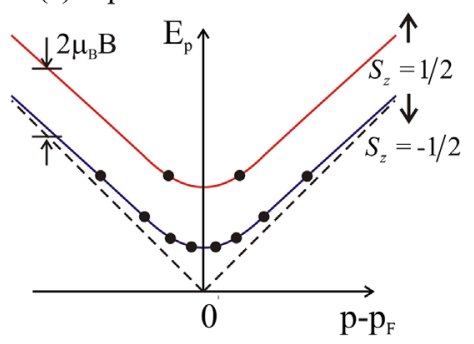

(b) $f_{T}$

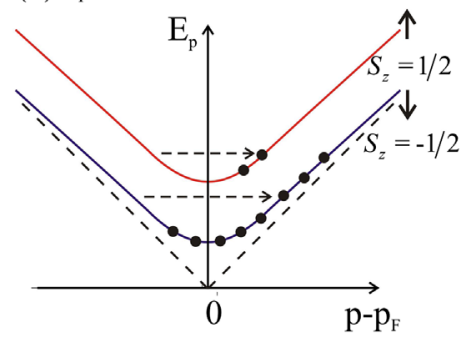

(c) $\mathrm{f}_{\mathrm{T}}$

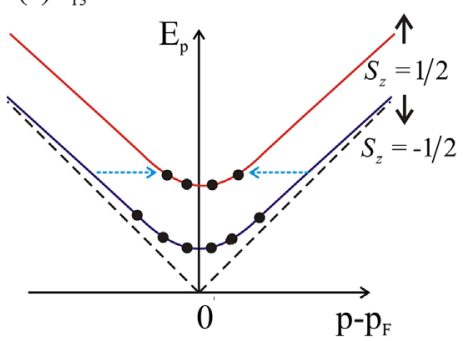

(d) $f_{\text {L3 }}$

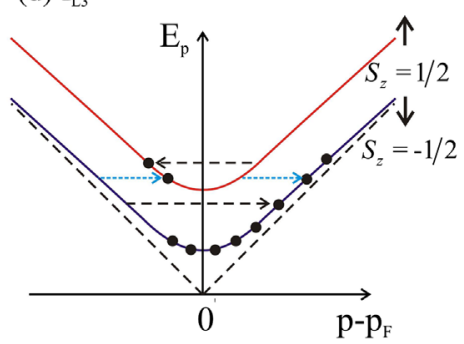

(e) $f_{L}$

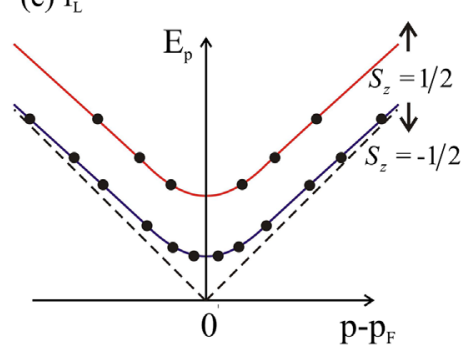

FIG. 4. Schematic of the quasiparticle distribution function components in a superconductor with spin splitting $2 \mu_{B} B$. The occupied states are represented by filled circles. (a) Equilibrium distribution, (b) electron-hole imbalance $f_{T}$, (c) spin imbalance $f_{T 3}$, (d) spinenergy imbalance $f_{L 3}$, and (e) energy imbalance $f_{L}$. The dashed and dotted arrows show elastic processes which lead to the formation, and the reverse processes to the relaxation, of a particular nonequilibrium mode. In (c) and (d) the dashed black lines show particle-hole branch transitions while the dotted blue lines correspond to the spin-flip processes. 
their couplings are at the basis of the main effects discussed in this Colloquium.

\section{A. Description of nonequilibrium modes in superconductors with spin splitting}

At this point we combine the pictorial description of the nonequilibrium modes (Fig. 4) with the quasiclassical formalism introduced in Sec. II.A and, in particular, the Usadel equation. For a description of nonequilibrium properties we need to consider the Keldysh component $\hat{g}^{K}$ of the quasiclassical GF [Eq. (2)]. For clarity we first consider a unique spin polarization direction parallel to the $z$ axis. From the normalization condition, Eq. (3), $\hat{g}^{K}$ can be expressed in terms of the retarded and advanced components and the generalized matrix distribution function $\hat{f}$ (Langenberg and Larkin, 1986):

$$
\hat{g}^{K}=\hat{g}^{R} \hat{f}-\hat{f} \hat{g}^{A}
$$

In the case of only one spin polarization axis, the $4 \times 4$ matrix distribution function $\hat{f}$ can be written as the sum of four components $^{2}$

$$
\hat{f}=f_{L} \hat{1}+f_{T} \tau_{3}+\left(f_{T 3} \sigma_{3}+f_{L 3} \sigma_{3} \tau_{3}\right) .
$$

For historical reasons we use the labeling introduced by Schmid and Schön (1975), generalized for the spin-dependent case. The $L$-labeled functions describe longitudinal modes, the spin energy degrees of freedom, and are antisymmetric in energy with respect to the Fermi level $\varepsilon=0$. The $T$-labeled functions describe transverse modes and are symmetric in energy. In equilibrium, the distribution function is proportional to the unit matrix in Nambu and spin space and given by

$$
\hat{f}_{\text {eq }}(\varepsilon)=\left(1-2 n_{F}\right) \hat{1}=\tanh (\varepsilon / 2 T) \hat{1} \text {. }
$$

We can now turn to the pictorial description of Fig. 4 and associate each component of $\hat{f}$ in Eq. (10) with a nonequilibrium mode as discussed next.

As shown in Figs. 4(b)-4(e), two of these modes have electron-hole branch imbalance, $f_{T}$ and $f_{L 3}$, while $f_{T 3}$ and $f_{L}$ are particle-hole symmetric. The filled circles in Fig. 4 represent the occupied states. As a reference, Fig. 4(a) corresponds to the equilibrium distribution function $\hat{f}=f_{L}^{0} \hat{1}=\tanh (\varepsilon / 2 T) \hat{1}$. In order to excite the nonequilibrium modes $f_{T}, f_{T 3}$ and $f_{L 3}$, one needs to move only the populated states (filled circles) between the different spectral branches in an elastic process, i.e., between equal-energy states (marked by horizontal dashed arrows). These modes can also relax back to equilibrium due to elastic scattering processes. The relaxation mechanisms depend on intrinsic material properties, degree and type of disorder, and also on the superconducting spectrum and are discussed in more detail in Sec. IV.

\footnotetext{
${ }^{2}$ Here we assume a unique spin polarization direction. In the most general case the distribution function has all spin components $\hat{f}=f_{L} \hat{1}+f_{T} \tau_{3}+\sum_{j}\left(f_{T j} \sigma_{j}+f_{L j} \sigma_{j} \tau_{3}\right)$.
}

The last nonequilibrium mode, the deviation of $f_{L}$ from $f_{L}^{0}$, is characterized by a change in the total quasiparticle number and energy content, corresponding to an increase or decrease of the effective temperature. It can be excited by increasing the number of occupied states to higher energies, and its relaxation requires inelastic processes.

In the absence of spin splitting, the charge imbalance is determined by $f_{T}$, and the energy imbalance by $f_{L}$. The spin splitting changes the system properties, mixing the coupling between spin-dependent modes and physical observables [see Eqs. (16) and (17)]. Qualitatively, the outcome can be seen by counting the number of occupied states on the different branches in Fig. 4. For example, the charge imbalance $\mu$ is determined by the difference between the number of occupied states in the electron and hole branches. Both $f_{T}$ and $f_{L 3}$ components contribute to it, as seen in Figs. 4(b) and 4(d).

On the other hand, a nonzero spin accumulation $\mu_{z}$ can be induced by exciting the modes $f_{T 3}$ or $f_{L}$ [Figs. 4(c) and 4(e)]. These two contributions to the total spin accumulation have important differences: The mode $f_{T 3}$ contributes to spin imbalance also in the absence of spin splitting. Spin imbalance in this mode can be induced, for example, by a spin-polarized injection from a ferromagnetic electrode in both the normal and the superconducting state. The relaxation of the spin accumulation created in this way is determined by elastic scattering processes. The second mechanism of inducing spin accumulation is by exciting the longitudinal mode $f_{L}$, in the presence of spin splitting [Fig. 4(e)]. Since energy-conserving transitions do not result in the relaxation of the $f_{L}$ mode, this component of the spin imbalance is not suppressed by elastic scattering. In other words, its relaxation can be provided only by inelastic processes, e.g., electron-phonon and electronelectron scattering. This result, obtained here on a phenomenological level, is crucial in understanding the long-range spin signal observed in superconductors, for example, by Hübler et al. (2012) and discussed in the next sections.

\section{B. Accumulations in terms of the nonequilibrium modes}

Quantitatively, we define the charge and spin accumulations based on the Keldysh component of the GF, Eq. (9),

$$
\begin{gathered}
\mu(\mathbf{r}, t)=-\int_{-\infty}^{\infty} \frac{d \varepsilon}{16} \operatorname{Tr} \hat{g}^{K}(\varepsilon, \mathbf{r}, t), \\
\mu_{\mathrm{sa}}(\mathbf{r}, t)=\int_{-\infty}^{\infty} \frac{d \varepsilon}{16} \operatorname{Tr} \tau_{3} \sigma_{a}\left[\hat{g}_{\mathrm{eq}}^{K}(\epsilon, \mathbf{r}, t)-\hat{g}^{K}(\varepsilon, \mathbf{r}, t)\right],
\end{gathered}
$$

whereas the local energy and spin-energy accumulations are given by

$$
\begin{array}{r}
q(\mathbf{r}, t)=\int_{-\infty}^{\infty} \frac{d \varepsilon}{16} \varepsilon \operatorname{Tr} \tau_{3}\left[\hat{g}_{\mathrm{eq}}^{K}(\varepsilon, \mathbf{r}, t)-\hat{g}^{K}(\varepsilon, \mathbf{r}, t)\right], \\
q_{\mathrm{sa}}(\mathbf{r}, t)=\int_{-\infty}^{\infty} \frac{d \varepsilon}{16} \varepsilon \operatorname{Tr} \sigma_{a}\left[\hat{g}_{\mathrm{eq}}^{K}(\varepsilon, \mathbf{r}, t)-\hat{g}^{K}(\varepsilon, \mathbf{r}, t)\right] .
\end{array}
$$

Here $a=1,2,3$ denotes the polarization direction of the nonequilibrium spins and energy is counted with respect to the potential $\mu_{S}$ of the superconducting condensate [see below Eq. (19)]. 
In terms of the distribution functions, the charge and spin accumulations read (we assume magnetization in the $z$ direction)

$$
\begin{gathered}
\mu=-\frac{1}{2} \int_{-\infty}^{\infty} d \varepsilon\left(N_{+} f_{T}+N_{-} f_{L 3}\right), \\
\mu_{z}=-\frac{1}{2} \int_{-\infty}^{\infty} d \varepsilon\left[N_{+} f_{T 3}+N_{-}\left(f_{L}-f_{\mathrm{eq}}\right)\right],
\end{gathered}
$$

where $N_{+}=N_{\uparrow}+N_{\downarrow}$ is the total DOS, $N_{-}=N_{\uparrow}-N_{\downarrow}$ is the DOS difference between the spin subbands present only when $h \neq 0$, and $f_{\text {eq }}(\varepsilon)=\tanh (\varepsilon / 2 T)$ is the equilibrium distribution function. Similarly for Eqs. (14) and (15) we get

$$
\begin{gathered}
q=\frac{1}{2} \int_{-\infty}^{\infty} d \varepsilon \varepsilon\left[N_{+}\left(f_{L}-f_{\text {eq }}\right)+N_{-} f_{T 3}\right], \\
q_{\mathrm{sa}}=\frac{1}{2} \int_{-\infty}^{\infty} d \varepsilon \varepsilon\left[N_{-}\left(f_{L}-f_{\mathrm{eq}}\right)+N_{+} f_{T 3}\right] .
\end{gathered}
$$

All these quantities Eqs. (12)-(19) are directly related to experimental observables. The charge imbalance $\mu$ characterizes the potential of the quasiparticles in the superconductor (Artemenko and Volkov, 1979). In nonequilibrium situations, $\mu$ can differ from the condensate potential $\mu_{S}$. In the problems discussed in this Colloquium, $\Delta$ can be chosen as time independent and $\mu_{S}=0$. The charge density depends on $\mu$ via $\rho=-\nu_{F} e^{2} \phi-e \nu_{F} \mu$, where $\phi$ is the electrostatic scalar potential (Kopnin, 2001). In metals, local charge neutrality is maintained on length scales large compared to the ThomasFermi screening length, so that $-e \phi=\mu$ and charge imbalance is associated with static electric fields.

In the quasiclassical formulation used here, electrochemical potential differences appear explicitly in energy shifts in the boundary conditions for the distribution functions (Belzig et al., 1999). The Fermi distribution at potential $V$ corresponds to

$f_{\mathrm{eq}, L(T)}(E)=\frac{1}{2}\left[\tanh \left(\frac{E+e V}{2 T}\right)+(-) \tanh \left(\frac{E-e V}{2 T}\right)\right]$.

For a superconductor in equilibrium, $V=0$ in this description. However, $V=\phi \neq 0$ can describe voltage-biased normal $(\Delta=0)$ reservoirs.

Spin accumulation is a standard observable in spintronics (Johnson and Silsbee, 1985; Jedema et al., 2002). The local energy accumulation is typically measured via electron thermometry (Giazotto et al., 2006). The spin-energy accumulation was measured recently in normal-state nanopillar spin valves (Dejene et al., 2013). To our knowledge this quantity has not been directly studied experimentally in superconducting systems.

In the normal state the spectrum is trivial, $g^{R(A)}= \pm \tau_{3} \sigma_{0}$. Thus, according to Eq. (9), the Keldysh component is simply proportional to the distribution function. In other words, the different modes decouple in Eqs. (12)-(19). Moreover, in the normal state it is unnecessary to separate between transverse and longitudinal modes and rather consider the spin-dependent full distribution function $f_{j}(\varepsilon)=\left[1-f_{L j}(\varepsilon)-f_{T j}(\varepsilon)\right] / 2$.
Solutions of the kinetic equation in the normal state are discussed, for example, by Brataas, Bauer, and Kelly (2006).

In the superconducting case the situation is more complex. First, the spectrum is strongly energy dependent around the Fermi level and the spectral GFs have a nontrivial structure in spin space. Components proportional to the unit matrix in spin space describe the BCS singlet GFs, whereas terms proportional to the Pauli matrices $\sigma_{j}, j=1,2,3$, describe the triplet state (Bergeret, Volkov, and Efetov, 2001b, 2005). Second, due to this energy dependence and nontrivial spin structure, the spectral functions enter Eqs. (12)-(15) and lead to a coupling between the different nonequilibrium modes that in turn couple all electronic degrees of freedom as discussed next.

\section{SPIN INJECTION AND DIFFUSION IN SUPERCONDUCTORS}

Nonequilibrium modes can be experimentally studied by means of nonlocal transport measurements. In this section we review experiments on charge and spin injection in superconductors and apply the kinetic equation approach described in the previous sections to describe different experimental situations.

\section{A. Detection of spin and charge imbalance: Nonlocal transport measurements}

Studies of the nonequilibrium modes started with the pioneering experiment of Clarke (1972), who realized a way of detecting the charge imbalance in a superconductor. The main idea of this experiment is to inject a current from a normal metal (injector) into a superconductor. This current creates a charge imbalance that corresponds to a shift of the chemical potential of the quasiparticles with respect to the one of the condensate. This shift of the chemical potential can be detected by a second electrode (detector) that probes the voltage between the superconductor and the detector.

More recent experiments used the same nonlocal measurement to explore the charge, energy, and spin modes in mesoscopic superconducting lateral structures (Beckmann, Weber, and Löhneysen, 2004; Poli et al., 2008; Hübler et al., 2010; Quay et al., 2013; Wolf et al., 2013; Wolf, Hübler et al., 2014). A scanning electron microscopy image of such a lateral structure is shown in Fig. 5. A detailed overview of the experiments on charge and energy imbalance can be found in the recent topical reviews by Beckmann (2016) and Quay and Aprili (2018).

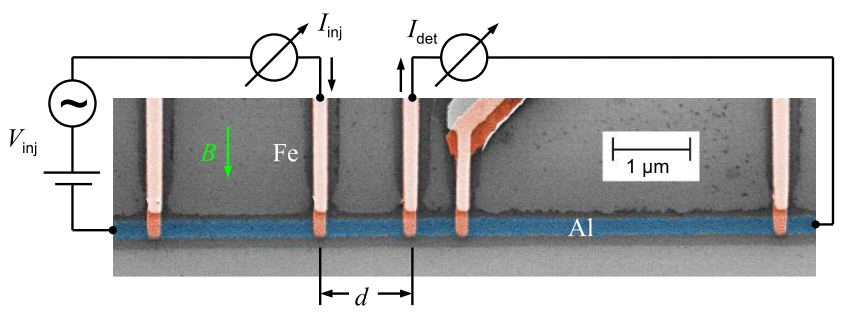

FIG. 5. Scanning electron microscopy image of the lateral structure. From Hübler et al., 2012. 
Whereas the charge and energy modes were known for a long time, it was first in the 1990s that theorists predicted how electronic charge and spin degrees of freedom can be separated in a superconductor (Kivelson and Rokhsar, 1990; Zhao and Hershfield, 1995). First experiments on F-S-F layered structures, however, did not show any evidence of such a spin-charge separation (Johnson, 1994; Gu et al., 2002) and the different relaxation times for spin and charge accumulation in superconductors remained an open question.

First clear insight into the separation of the spin and charge modes was obtained in experiments using lateral nanostructures with ferromagnetic injectors and detectors (Beckmann, Weber, and Löhneysen, 2004; Shin, Lee, and Lee, 2005; Cadden-Zimansky, Jiang, and Chandrasekhar, 2007; Poli et al., 2008; Yang et al., 2010; Hübler et al., 2012; Quay et al., 2013; Wolf et al., 2013; Wolf, Sürgers et al., 2014; Kolenda, Wolf, and Beckmann, 2016). First theoretical works on spin injection into mesoscopic superconductors (Morten, Brataas, and Belzig, 2004, 2005) showed that the spinrelaxation length in the superconducting state strongly depends on the energy of the injected quasiparticles and on the spin-relaxation mechanism. In particular, for a dominating spin-orbit scattering, superconductivity suppresses the spinrelaxation rate $\tau_{s}^{-1}$, which can be qualitatively understood as the decrease in the cross section of the quasiparticle momentum scattering at the energies near the gap edge $\varepsilon \sim \Delta$. The suppression of $\tau_{s}^{-1}$ is however compensated by the decrease in the quasiparticle group velocity $v_{g} \sim v_{F} \sqrt{1-|\Delta|^{2} / \varepsilon^{2}}$ so that the spin-relaxation length $\lambda_{\text {so }} \sim v_{g} \tau_{s}$ remains almost unchanged in the superconducting state. On the contrary, if the spin-flip mechanism dominates, the spin relaxation is not related to the momentum scattering because the interaction with magnetic impurities does not depend on the propagation direction and the quasiparticle spin does not depend on energy. This results in an increase of $\tau_{s}^{-1}$ which is equivalent to a decrease of the spin-relaxation length in the superconducting state. Although these works provided an explanation to some experiments, two important features observed in subsequent works could not be explained in terms of that theory: First, the spin accumulation was detected at distances from the injector much larger than the spin-relaxation length measured in the normal state (Hübler et al., 2012; Quay et al., 2013; Wolf et al., 2013). Second, an unexpected spin accumulation was observed even if the current was injected from a nonmagnetic electrode (Wolf et al., 2013). In order to explain these two observations one needs to take into account the spin splitting in the superconductor.

\section{B. Nonlocal conductance measurements in spin-split superconductors}

Specifically, one of the setups studied by Hübler et al. (2012) was a lateral nonlocal spin valve (see Fig. 5) where the experimentalists determined the nonlocal differential conductance

$$
g_{\mathrm{nl}}=\frac{d I_{\mathrm{det}}}{d V_{\mathrm{inj}}} .
$$
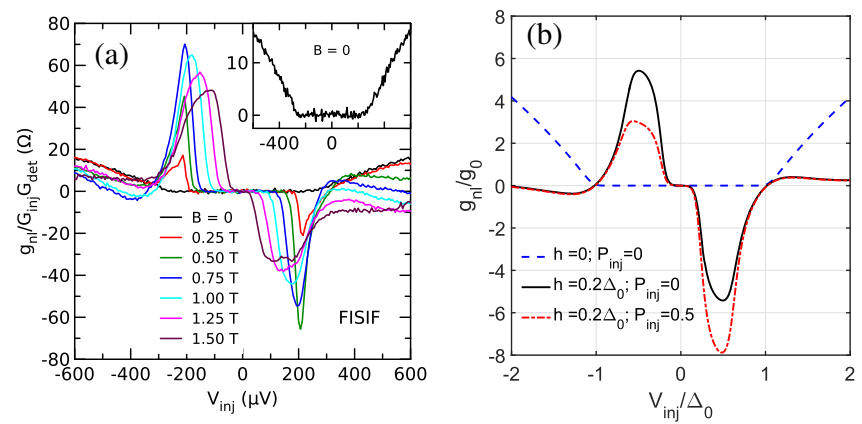

FIG. 6. (a) Nonlocal conductance measured as a function of the injecting voltage $g_{\mathrm{nl}}\left(V_{\mathrm{inj}}\right)$. Adapted from Hübler et al., 2012. (b) The same quantity calculated using the kinetic theory for $\alpha_{\text {orb }}=1.33, \beta=0.5,\left(\tau_{\mathrm{sn}} T_{c 0}\right)^{-1}=0.2, T=0.05 T_{c 0}$, effective inelastic relaxation length $L=20 \lambda_{\mathrm{sn}}, d=5 \lambda_{\mathrm{sn}}$, where $\lambda_{\mathrm{sn}}=$ $\sqrt{D \tau_{\mathrm{sn}}}$ is the normal-state spin relaxation length. Solid black and dash-dotted red curves correspond to the injection from nonferromagnetic $\left(P_{\text {inj }}=0\right)$ and ferromagnetic $\left(P_{\text {inj }}=0.5\right)$ electrodes, respectively, at the spin splitting $h=0.2 \Delta_{0}$. The dashed blue line corresponds to $h=0$. The conductance is normalized to $g_{0}=R_{\xi} / R_{\text {inj }} R_{\text {det }}$, where $R_{\xi}=\xi / A_{s} \sigma_{N}$ is the normal-state resistance of the wire with length $\xi$ and cross section $A_{s}$.

Typical experimental curves are shown in Fig. 6(a) and Fig. 6(b) shows the results calculated from the kinetic equations.

If the detector is a ferromagnet with magnetization collinear with the spin accumulation in the wire, the current at the detector for $V_{\text {det }}=0$ is obtained from Eqs. (7) and (8),

$$
I_{\mathrm{det}}=\left(\mu+P_{\mathrm{det}} \mu_{z}\right) / R_{\mathrm{det}},
$$

where $R_{\mathrm{det}}=R_{\square} / A$ is the detector interface resistance in the normal state, $A$ is the cross-sectional area of the detector, $\mu$ is the charge imbalance, and $\mu_{z}$ is the spin imbalance defined in Eqs. (12) and (13). According to the explicit expressions (16) and (17), the full description of the nonlocal current requires all four nonequilibrium modes.

Particularly interesting is the contribution from the second term on the rhs of Eq. (17). It is nonzero when the spin splitting described by $N_{-}$is nonzero and it provides a qualitative explanation of the experiments by Hübler et al. (2012), Quay et al. (2013), and Wolf et al. (2013): The spin imbalance $\mu_{z}$, being related to the energy nonequilibrium mode $f_{L}$, once excited can relax only via inelastic processes, especially mediated by the electron-phonon interaction. At low temperatures the corresponding decay length can be much larger than the spin decay length in normal metals. This explains the long-range nonlocal signal observed in the experiments. The observed long-range spin accumulation can thus be understood to result from the spin accumulation generated by the effective heating of the superconducting wire caused by the injection of nonequilibrium quasiparticles with energies larger than the superconducting gap (Bobkova and Bobkov, 2015, 2016; Krishtop, Houzet, and Meyer, 2015; Silaev, Virtanen, Bergeret, and Heikkilä, 2015; Silaev, Virtanen, Heikkilä, and Bergeret, 2015; Virtanen, Heikkilä, and Bergeret, 2016). Such a heating can originate, for example, by an injected current even from the 
nonferromagnetic electrode. The heating is not sensitive to the sign of the bias voltage at the injector and hence the generated spin imbalance must be an even function of the voltage $\mu_{z}\left(V_{\text {inj }}\right)=\mu_{z}\left(-V_{\text {inj }}\right)$. This leads to an antisymmetric shape of the nonlocal spin signal in $g_{\mathrm{nl}}$ with respect to $V_{\mathrm{inj}}$, in agreement with the experimental observation (Wolf, Hübler et al., 2014). All these features occur only if the superconductor has a spin-split density of states induced either by an external magnetic field or by the proximity to a ferromagnetic insulator.

A quantitative description of these effects can be provided by solving the kinetic equations for superconductors with spin-split subbands (Silaev, Virtanen, Bergeret, and Heikkilä, 2015). In this case the diffusion couples nonequilibrium modes pairwise. In particular, the kinetic equation (5) takes the form

$$
\nabla \cdot\left(\begin{array}{c}
j_{e} \\
j_{s} \\
j_{c} \\
j_{\mathrm{se}}
\end{array}\right)=\left(\begin{array}{cccc}
0 & 0 & 0 & 0 \\
0 & 0 & 0 & S_{T 3} \\
0 & 0 & R_{T} & R_{L 3} \\
0 & 0 & R_{L 3} & R_{T}+S_{L 3}
\end{array}\right)\left(\begin{array}{c}
f_{L} \\
f_{T 3} \\
f_{T} \\
f_{L 3}
\end{array}\right),
$$

where the spectral energy $j_{e}$, spin $j_{s}$, charge $j_{c}$, and spin energy $j_{\text {se }}$ currents derived from the general equation (6) are

$$
\left(\begin{array}{c}
j_{e} \\
j_{s} \\
j_{c} \\
j_{\mathrm{se}}
\end{array}\right)=\left(\begin{array}{cccc}
D_{L} & D_{T 3} & 0 & 0 \\
D_{T 3} & D_{L} & 0 & 0 \\
0 & 0 & D_{T} & D_{L 3} \\
0 & 0 & D_{L 3} & D_{T}
\end{array}\right)\left(\begin{array}{c}
\nabla f_{L} \\
\nabla f_{T 3} \\
\nabla f_{T} \\
\nabla f_{L 3}
\end{array}\right) .
$$

Here $D_{L / T / T 3 / L 3}$ are kinetic coefficients related to the spectral GFs (Silaev, Virtanen, Bergeret, and Heikkilä, 2015), $S_{T 3 / L 3}$ are parts of collision integrals describing spin relaxation, and $R_{T / L 3}$ is the coupling between the quasiparticles and the superconducting condensate.

On the one hand, the charge is coupled to the $f_{T}$ and $f_{L 3}$ modes [lower right block of Eq. (24)]. The relaxation of both of these modes, the right-hand side of Eq. (23), is nonvanishing for all energies, below and above the gap due to the magnetic pair breaking effects (Schmid and Schön, 1975; Nielsen et al., 1982). On the other hand, the spin-splitting field couples the spin and energy modes $f_{T 3}$ and $f_{L}$, respectively [upper left block of Eq. (24)]. As explained above, the energy mode can decay only via inelastic scattering which at low temperature can be disregarded compared to the spin relaxation.

Solutions of Eqs. (23) and (24) along with Eqs. (16) and (22) reproduce the main features of the measured nonlocal conductance presented in Fig. 6(a). Depending on the magnitudes of the spin-splitting field $h$ and the injector polarization $P_{\text {inj }}$, we can identify three distinct parameter regimes affecting the symmetry of $g_{\mathrm{nl}}$. (i) When $h=P_{\text {inj }}=0$ [dashed blue curve in Fig. 6(b)], the only contribution to the detector current comes from charge imbalance and $g_{\mathrm{nl}}$ is a symmetric function of the injection voltage. In the absence of spin splitting and depairing effects, $R_{T}=0$ for $\varepsilon>\Delta$, and hence charge imbalance decays only via inelastic scattering neglected here. This explains the monotonic increase of $g_{\mathrm{nl}}$ in Fig. 6(b) at large voltages. (ii) For $P_{\text {inj }}=0$ but in the presence of an applied field leading to $h \neq 0$ (solid black curve), charge relaxation is strongly enhanced due to the orbital depairing. The main long-range contribution comes from $\mu_{z}$ produced by the heating effect described in the beginning of this section. The resulting $g_{\mathrm{nl}}$ is an antisymmetric function of $V_{\text {inj. }}$ (iii) When both $h \neq 0$ and $P_{\text {inj }} \neq 0$ (dashdotted red curve), an additional symmetric long-range contribution in $g_{\mathrm{nl}}$ results due to a thermoelectric effect at the injector. Note that in the case $h=0, P_{\text {inj }} \neq 0$, there would be another symmetric contribution to $g_{\mathrm{nl}}$ due to the regular spin injection also present in the normal state. However, this is a short-range mode (decays via spin relaxation) and therefore does not show up beyond the spin-relaxation length.

In the experiments by Hübler et al. (2012), Quay et al. (2013), and Wolf, Hübler et al. (2014) the spin-splitting field was caused by an external magnetic field. Therefore one needs to take into account the orbital depairing effect of the magnetic field in addition to the Zeeman effect. The relative strength of the orbital depairing and the spin-splitting field is described by the dimensionless parameter $\alpha_{\text {orb }}=\left(h \tau_{\text {orb }}\right)^{-1}$. In Fig. 6 we choose the value $\alpha_{\text {orb }}=1.33$, which should correspond to the experiment by Hübler et al. (2012).

In the presence of a supercurrent, all coefficients of the matrix in Eq. (24) are nonzero (Aikebaier, Silaev, and Heikkilä, 2018). As a result, for example, the spin and charge modes are directly coupled by diffusion.

\section{Spin Hanle effect}

In the previous sections we assume that all magnetizations and the applied field are collinear. If one lifts this assumption, the applied field leads to a precession of the injected spin around the field direction. This is the spin Hanle effect that in the normal state has been extensively studied in the literature and observed in several experiments (Johnson and Silsbee, 1985; Jedema, Filip, and van Wees, 2001; Jedema et al., 2003; Yang, Kimura, and Otani, 2008; Villamor et al., 2015). The Hanle precession can be measured via the nonlocal conductance in a setup such as the one shown in Fig. 5. The nonlocal measured signal oscillates and decays as a function of the amplitude of the applied field.

Formally the Hanle effect is described by the first term on the rhs of Eq. (5). Indeed, one can derive the Bloch-Torrey transport equation (Torrey, 1956) for the magnetic moment $\boldsymbol{m}(\varepsilon, x)=\operatorname{Tr}\left(\tau_{3} \boldsymbol{\sigma} g^{K}\right) / 8$ from Eq. (5) (Silaev, Virtanen, Heikkilä, and Bergeret, 2015). It reads

$$
\frac{\partial \boldsymbol{m}}{\partial t}+\nabla \cdot \boldsymbol{j}_{S}=\gamma \boldsymbol{m} \times \boldsymbol{h}_{s}-\boldsymbol{m} / \tau_{S} .
$$

Here $\gamma=-2$ is the electron gyromagnetic ratio and $\boldsymbol{j}_{s}$ is the spin current density tensor. In the normal state the spin relaxation $\tau_{S}$ and Zeeman field $\boldsymbol{h}_{s}$ are energy independent. This explains why the nonlocal resistance versus the field curve does not depend on either the temperature or the type of spin relaxation (magnetic or spin-orbit impurities). In contrast, they are predicted to be strongly energy dependent in the superconducting state, and the precession and decay of the nonlocal signal disappear at $T \rightarrow 0$, whereas the shape of 
the curves at intermediate temperatures depends on the type of spin relaxation (Silaev, Virtanen, Heikkilä, and Bergeret, 2015). Experimental evidence of the Hanle effect in the superconducting state has not been reported so far.

\section{Spin imbalance by ac excitation}

The quasiparticle $f_{T, j}$ mode-or, equivalently, the quasiparticle magnetic moment $\boldsymbol{m}(\varepsilon, x)$-can be excited by an external ac magnetic field, which via the Zeeman coupling generates a conduction electron spin resonance (Aoi and Swihart, 1970; Maki, 1973; Vier and Schultz, 1983; Yafet, Vier, and Schultz, 1984; Nemes et al., 2000). This was recently studied experimentally in spin-split thin $\mathrm{Al}$ films by Quay et al. (2015). As the $f_{T, j}$ mode can relax rapidly via elastic spin-flip scattering, the linewidth seen in such experiments is generally $\tau_{S}^{-1} \simeq \tau_{\mathrm{sn}}^{-1}$ instead of the time scale of the long-ranged nonlocal spin signal. Spin-flip scattering also provides a channel via which electromagnetic fields can generate spin imbalance through the orbital coupling (van Bentum and Wyder, 1986; Virtanen, Heikkilä, and Bergeret, 2016). For high enough driving amplitude, the imbalance modifies the self-consistent $\Delta(T)$ relation, which develops additional features in the spin-split case (Eliashberg, 1970; Virtanen, Heikkilä, and Bergeret, 2016). Effects related to spin splitting and relaxation can moreover be probed with tunnel junctions at low frequencies (Quay et al., 2016) or via photoassisted tunneling (Marchegiani et al., 2016).

\section{THERMOELECTRIC EFFECTS}

Thermoelectric effects relate temperature differences to charge currents and electrical potentials to heat currents. Thermoelectric effects are typically described via the linear-response relation between charge and heat currents $I, \dot{Q}$ and bias voltage and temperature difference $V$ and $\Delta T$ across a contact $^{3}$ :

$$
\left(\begin{array}{c}
I \\
\dot{Q}
\end{array}\right)=\left(\begin{array}{cc}
G & \alpha \\
\alpha & G_{\mathrm{th}} T
\end{array}\right)\left(\begin{array}{c}
V \\
-\Delta T / T
\end{array}\right)
$$

Here $G$ is the conductance and $G_{\text {th }}$ the heat conductance of the contact. $\alpha$ is the thermoelectric coefficient.

With a nonzero $\alpha$, electrical energy may be converted to heat or cooling, or reciprocally a temperature difference may be converted to electrical power. The efficiency of this conversion is typically described by the thermoelectric figure of merit,

\footnotetext{
${ }^{3}$ In the case of thermoelectric effects, it is customary to discuss heat currents $\dot{Q}$ instead of energy currents $\dot{U}$, and we adapt this convention here. These are related by (Ashcroft and Mermin, 1976) $\dot{Q}=\dot{U}-\mu I / e$, where $\mu$ is a reference energy compared to the Fermi level. At linear response we can set $\mu=0$ in which case $\dot{Q}=\dot{U}$. On the other hand, when considering heat balance at nonvanishing voltages as in Sec. V.A, the two are not the same and rather the heat current $\dot{Q}$ should be used.
}

$$
Z T=\frac{\alpha^{2}}{G_{\mathrm{th}} G T-\alpha^{2}}=\frac{S^{2} G T}{\tilde{G}_{\mathrm{th}}},
$$

where $S=\alpha / G T$ is the thermopower (Seebeck coefficient) and $\tilde{G}_{\text {th }}=G_{\text {th }}-\alpha^{2} / G T$ is the thermal conductance at a vanishing current. In particular, the maximum efficiency of a thermoelectric heat engine is (Snyder and Ursell, 2003)

$$
\max \eta=\eta_{\text {Carnot }} \frac{\sqrt{1+Z T}-1}{\sqrt{1+Z T}+1}
$$

with $\eta_{\text {Carnot }}=\Delta T / T$. Maximum efficiencies of the device are obtained when $Z T \rightarrow \infty$. At or above room temperature, the record-high figures of merit are obtained in certain strongly doped semiconductor structures (Kim et al., 2015; Zhao et al., 2016). A typical record value for those cases is $Z T \gtrsim 1-2$.

The traditional view of thermoelectric effects in superconductors is that if they exist, they must be very weak. In bulk superconductors, this is partially because any thermoelectrically generated quasiparticle current is easily screened by a supercurrent (Meissner, 1927).

Alternatively, one could then measure this supercurrent via an additional constraint to the phase of the superconducting order parameter in bimetallic multiply connected structures (Ginzburg, 1944). However, even this thermally created phase gradient tends to be weak, owing to the near-complete electron-hole symmetry in superconductors. Galperin, Gurevich, and Kozub (1974) showed that

$$
\alpha=\alpha_{N} G(\Delta / T), \quad G(x)=\frac{3}{2 \pi^{2}} \int_{x}^{\infty} \frac{y^{2} d y}{\cosh ^{2}(y / 2)},
$$

where the latter form comes from the reduction of the quasiparticle density in the superconducting state, and $\alpha_{N}$ is the value of the thermoelectric coefficient in the normal state. The precise value of $\alpha_{N}$ depends on the exact electronic spectrum. For example, for a simple quadratic dispersion

$$
\alpha_{N}=\frac{\pi^{2} G_{T} k_{B}^{2} T}{6 e E_{F}}
$$

where $E_{F}$ is the Fermi energy. At temperatures $T \ll \Delta / k_{B}, \alpha$ is thus expected to be a product of two small coefficients $\alpha_{N} \propto k_{B} T / E_{F}$ and $G(\Delta / T)$. This is very small and not easy to measure quantitatively.

However, superconductors do contain some ingredients for strong thermoelectric effects, because the latter typically require strongly energy-dependent density of states of the charge carriers. This is provided by the BCS density of states. Hence, if one can break the electron-hole symmetry of the transport process via some mechanism, superconductors can become very strong thermoelectrics. This is precisely what happens in spin-split superconductors, as an exchange field breaks the symmetry in each spin sector, but so that the overall spin-summed energy spectrum remains electron-hole symmetric. Transport through a spin filter to a spin-split superconductor then can provide large thermoelectric effects because the two spins are weighed differently (Machon, Eschrig, and Belzig, 2013; Machon, Eschrig, and Belzig, 
2014; Ozaeta et al., 2014). We discuss these effects in this section.

\section{A. Charge and heat currents at a spin-polarized interface to a spin-split superconductor}

Consider a tunnel contact from a nonsuperconducting reservoir $R$ to a superconductor $S$ in a spin-splitting field. Let us assume that the tunnel contact is magnetic, so that the conductance through it is spin polarized. Denoting the spindependent conductances in the normal state by $G_{\uparrow}, G_{\downarrow}$ we can parametrize them by the total conductance $G_{T}=G_{\uparrow}+G_{\downarrow}$ and the spin polarization $P=\left(G_{\uparrow}-G_{\downarrow}\right) / G_{T}$. The total tunneling quasiparticle charge and heat currents are now expressed as a sum over spin-dependent contributions, but otherwise of the standard form (Giaever and Megerle, 1961; Giazotto et al., 2006). Denoting the spin-dependent reduced density of states via $N_{+}=N_{\uparrow}+N_{\downarrow}$ and $N_{-}=N_{\uparrow}-N_{\downarrow}$ the spin-averaged tunnel currents can be obtained from the Keldysh component of Eq. (8) after taking the corresponding traces:

$$
\begin{gathered}
I=\frac{G_{T}}{2 e} \int_{-\infty}^{\infty} d \varepsilon\left(N_{+}+P N_{-}\right)\left(f_{R}-f_{S}\right), \\
\dot{Q}_{i}=\frac{G_{T}}{2 e} \int_{-\infty}^{\infty} d \varepsilon\left(\varepsilon-\mu_{i}\right)\left(N_{+}+P N_{-}\right)\left(f_{R}-f_{S}\right) .
\end{gathered}
$$

Here $f_{R / S}=n_{F}\left(\varepsilon-\mu_{R / S} ; T_{R / S}\right), n_{F}(\varepsilon ; T)=\left\{\exp \left[\varepsilon /\left(k_{B} T\right)\right]+\right.$ $1\}^{-1}$ are the Fermi functions of the reservoirs biased at potentials $\mu_{R / S}$ and temperatures $T_{R / S}$. The reduced density of states in the superconductor for spin $\sigma$ is $N_{\sigma}(\varepsilon)$. The heat current $\dot{Q}_{\sigma}^{i}$ is calculated separately for $i=R, S$, using the potential $\mu_{R / S}$, because the two heat currents differ by the Joule power $I\left(\mu_{R}-\mu_{S}\right) / e$. In the following analysis, we disregard the spin-relaxation effects on the density of states, because this assumption allows for some analytically treatable limits and it is a fair approximation in the case of often used Al samples.

The heat current from $R$ is a nonmonotonous function of voltage even in the absence of spin polarization or temperature difference. In particular, for voltage $V=\left(\mu_{R}-\mu_{S}\right) \approx \Delta / e$, it is positive, i.e., reservoir $R$ cools down (Nahum, Eiles, and Martinis, 1994; Leivo, Pekola, and Averin, 1996; Pekola et al., 2004). This heat current is quadratic in the voltage, and therefore it does not result from the usual Peltier effect [Eq. (26) for $\dot{Q}$ ] where the cooling power is linear in voltage.

Interestingly, in the presence of spin polarization $P$ and with a nonzero spin-splitting field $h$ in the superconductor, the cooling power is nonzero even in the linear-response regime, i.e., low voltages (Ozaeta et al., 2014). As an example we show in Fig. 7 the cooling power from reservoir $R$ as a function of voltage for various values of $h$, assuming the ideal case of unit spin polarization $P=1$.

Contrary to the spin-independent case, the N-FI-S element can also be used to refrigerate the superconductor. Electron refrigeration using magnetic elements have been studied by Rouco, Heikkilä, and Bergeret (2018).

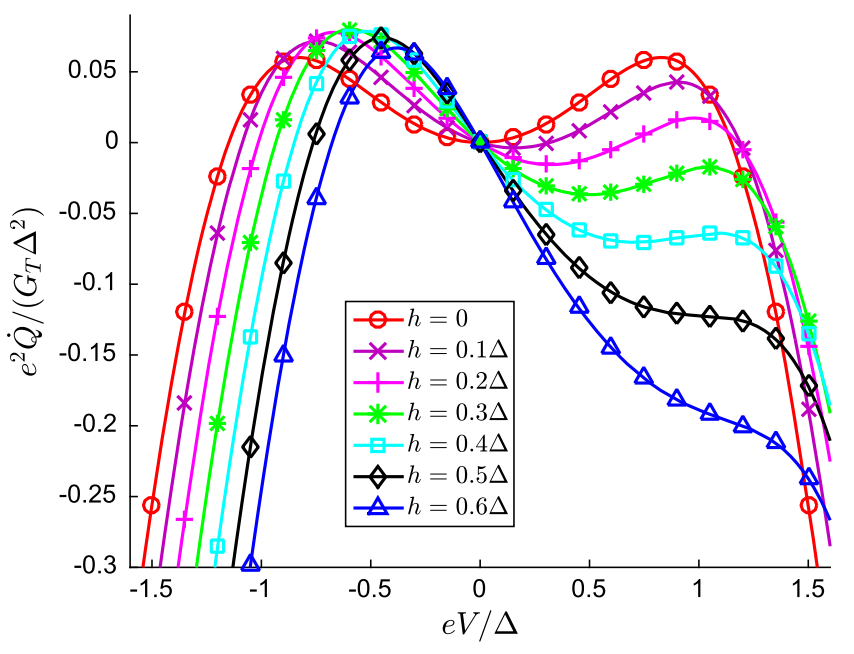

FIG. 7. Cooling power from reservoir $R$ vs voltage for different values of the exchange field $h$, assuming a unit polarization $P=1$ at the temperature $k_{B} T=0.3 \Delta$ close to that yielding optimal cooling for $P=h=0$. The exchange fields are given in units of $\Delta$. Changing the sign of $P$ or $h$ inverts the voltage dependence with respect to $V=0$.

\section{B. Linear response: Heat engine based on a superconductor/ferromagnet structure}

As can be seen in Fig. 7, the simultaneous presence of the nonvanishing spin polarization $P$ and a spin-splitting field $h$ leads to a heat current that has a linear component in the voltage $V$. This component is nothing else than the Peltier effect. In the limit $k_{B} T \ll \Delta-h$ the linear-response coefficients evaluate to (Ozaeta et al., 2014)

$$
\begin{gathered}
G \approx G_{T} \sqrt{2 \pi \tilde{\Delta}} \cosh (\tilde{h}) e^{-\tilde{\Delta}}, \\
G_{\text {th }} \approx \frac{k_{B} G_{T} \Delta}{e^{2}} \sqrt{\frac{\pi}{2 \tilde{\Delta}}} e^{-\tilde{\Delta}}\left[e^{\tilde{h}}(\tilde{\Delta}-\tilde{h})^{2}+e^{-\tilde{h}}(\tilde{\Delta}+\tilde{h})^{2}\right], \\
\alpha \approx \frac{G_{T} P}{e} \sqrt{2 \pi \tilde{\Delta}} e^{-\tilde{\Delta}}[\Delta \sinh (\tilde{h})-h \cosh (\tilde{h})],
\end{gathered}
$$

where $\tilde{\Delta}=\Delta / k_{B} T$ and $\tilde{h}=h / k_{B} T$. These yield the thermopower

$$
S=\frac{\alpha}{G T} \approx \frac{P \Delta}{e T}[\tanh (\tilde{h})-h / \Delta] .
$$

At low temperatures the thermopower is maximized for $h=k_{B} T \operatorname{arcosh}\left[\Delta /\left(k_{B} T\right)\right]$, where it is

$$
S_{\max } \approx \frac{k_{B}}{e} P\left[\frac{\Delta}{k_{B} T}-\operatorname{arcosh}\left(\sqrt{\frac{\Delta}{k_{B} T}}\right)\right] .
$$

It can hence become much larger than the "natural scale" $k_{B} / e$ and even diverge toward low temperatures. However, such a divergence comes together with the vanishing of the conductance, Eq. (31), and therefore is in practice cut off either by circuit effects, where the impedance to the volt meter becomes lower than the contact impedance, due to neglecting spin 
relaxation, or alternatively by additional contributions beyond the BCS model. The latter ones are described in more detail by Ozaeta et al. (2014). Nevertheless, with proper circuit design one should be able to measure a thermopower much exceeding $k_{B} / e$ in this setup.

These theoretical predictions in the linear-response regime were confirmed experimentally by Kolenda, Wolf, and Beckmann (2016) and Kolenda et al. (2017). In particular, they prepared a sample containing a crossing of three types of metals: a normal-metallic $\mathrm{Cu}$, a ferromagnetic $\mathrm{Fe}$, and a superconducting $\mathrm{Al}$. The measured configuration is sketched in Fig. 8(a). The electrons in the ferromagnetic wire were heated with the heater current $I_{\text {heat }}$, producing a temperature difference between the ferromagnet and the superconductor. The contact between the ferromagnet and the normal metal was Ohmic and therefore the temperature difference between them was negligibly small. Then the thermoelectric current was measured as a function of the magnetic field $\boldsymbol{B}$ applied parallel to the ferromagnetic wire. The agreement between the experimental results and the tunneling theory was excellent (see Fig. 9). The temperature difference between the ferromagnet and the superconductor was a fitting parameter, whereas the polarization $P$ was fitted from the conductance spectrum. In the experiment it was fitted to the value $P=0.08$, a modest value attributed to the thin oxide barrier between the $\mathrm{Fe}$ and the $\mathrm{Al}$ layers. In principle larger values of $P$ can be obtained by increasing the thickness of the oxide barrier (Münzenberg and Moodera, 2004), but this of course would reduce the amplitude of the thermoelectric current. (a)

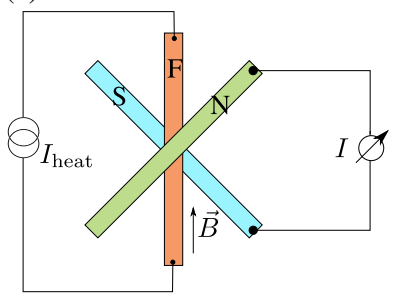

(c)

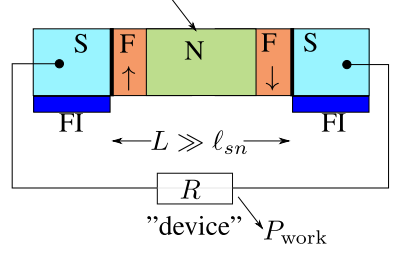

(b)

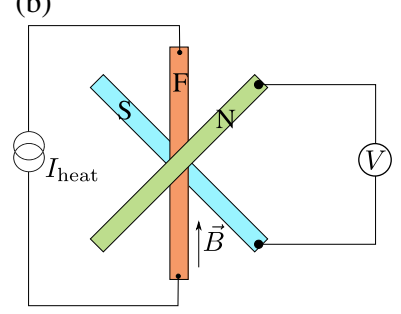

(d)

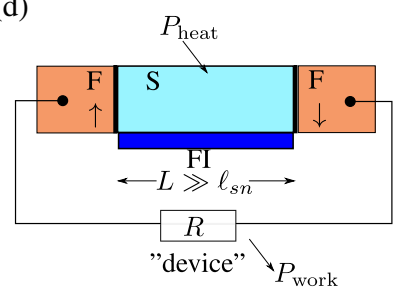

FIG. 8. (a) Schematic setup for measuring the thermoelectrically induced current, used by Kolenda, Wolf, and Beckmann (2016). $\mathrm{S}, \mathrm{F}$, and $\mathrm{N}$ stand for a superconductor, a ferromagnet, and a normal metal, whereas FI is a ferromagnetic insulator. (b) Setup used for a direct measurement of the Seebeck effect. (c) Heat engine realized in a lateral setup with " $n$-doped" and " $p$-doped" junctions using a FNF trilayer with antiparallel magnetization directions. To disregard spin accumulation, the island has to be large compared to the spin-relaxation length. (d) Heat engine with a spin-split superconducting island. The ferromagnets can also be replaced by a normal metal if the interfaces to the superconductor contain a ferromagnetic insulator. (c), (d) The heating power $P_{\text {heat }}$ is partially converted to "useful" work $P_{\text {work }}$ dissipated on the load.

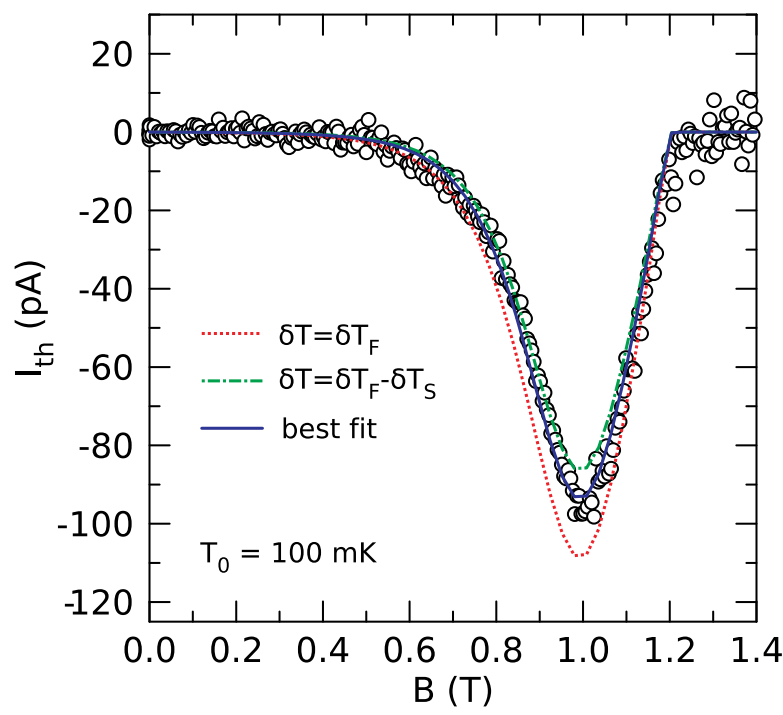

FIG. 9. Thermoelectric current as a function of the applied magnetic field, measured in Kolenda, Wolf, and Beckmann (2016). The circles show the measurement values, and the solid lines show a comparison to Eq. (29). The three solid lines correspond to slightly different temperature differences.

In the experiment, the thermoelectric current was measured rather than the voltage. In that case the impedance of the sample dominated that of the measurement lines. This is why the measurement yielded the exponentially low thermoelectric current, which nevertheless was sizable. The measurement configuration in Fig. 8(b) would have directly measured the generated voltage drop (i.e., Seebeck effect) instead of the current. This voltage results from the ratio of two exponentially small functions, the thermoelectric coefficient $\alpha$ and the conductance $G$, and itself is not small. Such a measurement would then tell about spurious effects, for example, due to spin relaxation, or due to the presence of fluctuations or states inside the gap. These effects would limit the diverging Seebeck coefficient at low temperatures (Ozaeta et al., 2014). Better still, replacing the normal metal with another superconductor with an inverse spin-splitting field would have resulted in twice as large a signal (corresponding to a series of $p$ - and $n$-doped thermoelectric elements), but would not be possible to create as such with a magnetic field. The solution would be furthermore to replace the ferromagnetic wire by an FNF heterostructure [Fig. 8(c), where the ferromagnets have antiparallel magnetizations, for example, due to different coercive fields, and the normal metal $\mathrm{N}$ would serve as a spacer between them]. To reach high figures of merit, the ferromagnetic metals should also be replaced by ferromagnetic insulators, which can reach very high values of spin polarization (see Table I), with $P$ exceeding 0.9 .

The island setup in Figs. 8(c) and 8(d) also realizes a thermally isolated structure, in contrast to those in Figs. 8(a) and $8(\mathrm{~b})$. This allows realizing a heat engine, where the voltage measurement is replaced by the "device" to be powered with the engine, with resistance that should be matched to the thermoelectric element. If only the electrons of the ferromagnetic island are heated, the main spurious heat 


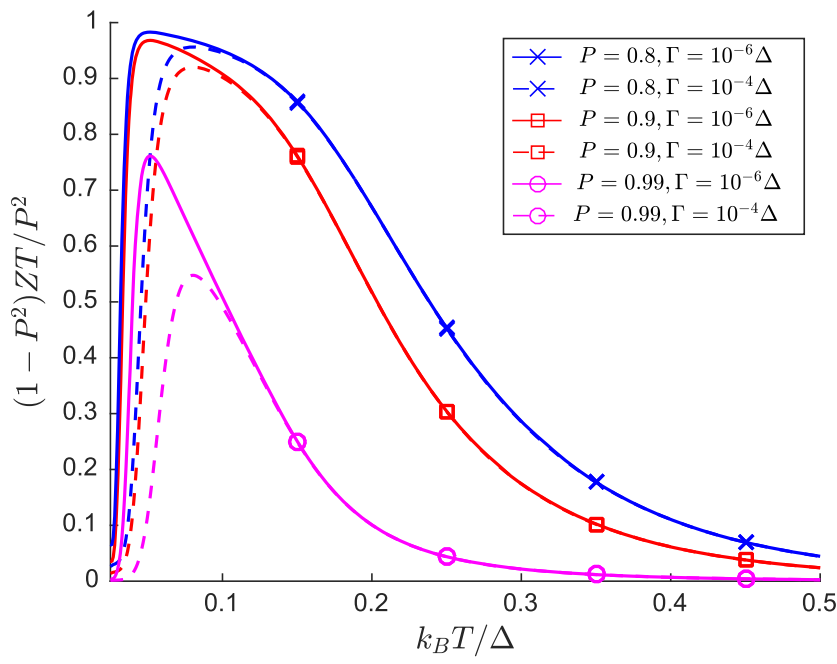

FIG. 10. Figure of merit in a N-FI-S-FI-N heat engine as a function of temperature for different polarizations $P$ of the junction. The figure has been calculated with $h=0.5 \Delta$ and $g=1000$, without calculating $\Delta$ self-consistently. The solid lines correspond to $\Gamma=10^{-6} \Delta$ and the dashed lines to $\Gamma=10^{-4} \Delta$. The figure of merit at low temperatures reaches very close to $P^{2} /\left(1-P^{2}\right)$ unless $P$ is very close to unity, but the exact temperature scale where this happens depends on the value of polarization. At the lowest temperatures $Z T$ is limited by another spurious heat conduction process, due to nonzero density of states inside the gap, described here by the Dynes $\Gamma$ parameter.

conduction mechanism is due to electron-phonon coupling. In that case it is advantageous to use the structure Fig. 8(d), because the electron-phonon heat conductance is weaker in a superconductor (Kaplan et al., 1976; Heikkilä et al., 2017) than in a normal metal (Wellstood, Urbina, and Clarke, 1994). For example, Fig. 10 shows a prediction for the resulting temperature dependence of the thermoelectric figure of merit $Z T$ in structure Fig. 8(d), including this spurious heat conduction. In an optimized structure, very large $Z T$ could thus be expected. In the picture, $g=5 k_{B}^{5} \sqrt{2 \pi} e^{2} \Sigma \Omega \Delta^{3} /\left(2 G_{T}\right)$ is a dimensionless quantity characterizing the relative strength of electron-phonon coupling (characterized by $\Sigma$ ) (Giazotto et al., 2006) to the tunnel coupling of the thermoelectric element in an island with volume $\Omega$. For example, for $\Omega=0.005 \mu \mathrm{m}^{3}, \Sigma=10^{9} \mathrm{~W} \mu \mathrm{m}^{-3} \mathrm{~K}^{-5}$ and $1 / G_{T}=30 \mathrm{k} \Omega$, $g=1000$.

Note that it is really the presence of the spurious electronphonon heat conduction that limits the highest available values of ZT. Often such spurious mechanisms are disregarded from the theoretical analysis, for example, in the case of quantum dots (Hwang, López, and Sánchez, 2016).

Even if the true figure of merit of this type of heat engine can be made high, these systems cannot obviously be used to replace room-temperature thermoelectric devices to be applied, for example, in energy harvesting. However, there are other applications where the large figure of merit may turn out to be essential. For example, this type of thermoelectric heat engine can be used for thermal radiation sensing at low temperatures (Giazotto et al., 2006; Heikkilä et al., 2017). Another possible use of the thermoelectric effects would be in noninvasive low-temperature thermometry (Giazotto et al., 2015), where the temperature (difference) profiles could be read from the thermopower without having to apply currents. In a scanning mode this would hence be a low-temperature version of the method used by Menges et al. (2016).

Note that this discussion disregards the effect of spin-orbit or spin-flip scattering on the superconducting state. It limits $Z T$ especially in heavy-metal superconductors. The associated effects were considered by Bergeret et al. (2017) and Rezaei et al. (2018).

\section{Spin Seebeck effect}

Besides the large thermoelectric effect, the contact between spin-split superconductors with other conducting materials can exhibit a large (longitudinal) spin Seebeck effect, where a temperature difference drives spin currents to and from the spin-split superconductor (Ozaeta et al., 2014). In this case the charge, heat, spin, and spin heat currents are described by the full (Onsager, 1931; Jacquod et al., 2012; Machon, Eschrig, and Belzig, 2013) Onsager linear-response matrix

$$
\left(\begin{array}{c}
I \\
\dot{Q} \\
I_{s} \\
\dot{Q}_{s}
\end{array}\right)=\left(\begin{array}{cccc}
G & \alpha & P G & \tilde{\alpha} \\
\alpha & G_{\mathrm{th}} T & \tilde{\alpha} & P G_{\mathrm{th}} T \\
P G & \tilde{\alpha} & G & \alpha \\
\tilde{\alpha} & P G_{\mathrm{th}} T & \alpha & G_{\mathrm{th}} T
\end{array}\right)\left(\begin{array}{c}
V \\
-\Delta T / T \\
V_{s} / 2 \\
-\Delta T_{s} / 2 T
\end{array}\right),
$$

where for $k_{B} T \ll \Delta-h$ the coefficients $G, G_{\text {th }}$, and $\alpha$ are given in Eqs. (31)-(33), and $\tilde{\alpha}=\alpha / P$. Here $V_{s}$ and $\Delta T_{s}$ refer to spin-dependent biases (Bergeret et al., 2017).

The spin currents induced in the case of two spin-split superconductors, and the additional effects of Josephson coupling, magnetization texture, and spin-orbit effects were discussed by Linder and Bathen (2016) and Bathen and Linder (2017). When either of the two materials realizes an island, the spin current can be converted into a spin accumulation $\mu_{z}$ that is determined from the balance between thermally induced spin currents and spin relaxation within the island. This discussion on heat engines assumes a structure size much longer than the spin-relaxation length and hence disregards this spin accumulation. The effect of the thermally induced spin accumulation on the superconducting gap was considered by Bobkova and Bobkov (2017), who predicted the associated changes in the critical temperature.

This spin Seebeck effect should be contrasted to the analogous phenomenon discussed in nonsuperconducting materials (Uchida et al., 2014). There, a major contribution to the spin Seebeck signal is due to the thermally induced spin pumping (Hoffman, Sato, and Tserkovnyak, 2013).

\section{Thermophase in a $S(F I) S$ contact}

The large thermoelectric effect allows for a large thermally induced phase gradient. This was theoretically investigated by Giazotto, Heikkilä, and Bergeret (2015). The total current in this case consists of the sum of a thermoelectric current $I_{\text {th }}$ and the supercurrent 


$$
I=I_{\mathrm{th}}+I_{c} \sin (\varphi),
$$

where $I_{\text {th }}$ is obtained from Eq. (29) and $I_{c}$ is the critical current for the junction with a phase difference $\varphi$ of the order parameters across the contact. The critical current is proportional to $\sqrt{1-P^{2}}$ (Bergeret, Verso, and Volkov, 2012) and depends on the spin-splitting field in $\mathrm{S}$ (Bergeret and Giazotto, 2014).

In an electrically open configuration, the two currents must cancel, and instead a thermophase $\varphi^{\text {th }}$ develops across the junction. This is obtained from

$$
\sin \left(\varphi^{\mathrm{th}}\right)=-\frac{I_{\mathrm{th}}}{I_{c}} .
$$

The thermophase can be detected using a bimetallic loop with two contacts, characterized by critical currents $I_{c 1,2}$ and thermophases $\varphi_{1,2}^{\text {th }}$. For nonzero exchange field and spin polarization $P$, the resulting thermophases can be much larger than in ordinary bulk superconductors. Hence the temperature dependence of the inductances play a more minor role than in the case of superconductors without spin splitting (Van Harlingen, Heidel, and Garland, 1980; Shelly, Matrozova, and Petrashov, 2016). For junctions with nonequal thermophases and for negligible loop inductance (in practice, $\left.2 e L I_{c 1,2} / \hbar \ll 1\right)$ in the absence of an external flux the circulating current is

$$
I_{\text {circ }}=\frac{I_{c 1} I_{c 2}}{I_{c 1}+I_{c 2}}\left[\sin \left(\varphi_{1}^{\mathrm{th}}\right)-\sin \left(\varphi_{2}^{\mathrm{th}}\right)\right] .
$$

In the case of symmetric junctions both thermophases are the same and the circulating current in the absence of an external flux vanishes. However, as discussed by Giazotto, Heikkilä, and Bergeret (2015), the thermoelectric current affects the response of the circulating current to the external flux, allowing for their measurement also in that case.

Equation (38) requires that both sides of the equation have an absolute value of at most unity, i.e., $\left|I_{\mathrm{th}}\right|<I_{c}$. For a very large thermoelectric current, its cancellation with a supercurrent is no longer possible, and instead a voltage across the contact forms. In this case the direct current response of the junction is more similar to the case discussed in the linearresponse limit for a N-FI-S junction. This regime was investigated in detail by Linder and Bathen (2016). Moreover, the nonvanishing dc voltage across the superconducting junction leads to Josephson oscillations at the frequency $2 \mathrm{eV} / \mathrm{h}$, where $h$ is the Planck constant. Hence, the device can be used as a temperature (difference) to frequency converter as discussed in more detail by Giazotto et al. (2015).

\section{SUMMARY AND OUTLOOK}

This Colloquium focuses on transport and thermal properties of superconducting hybrid structures with a spin-split density of states. Such a splitting can be achieved either by an external magnetic field or, more interestingly, by placing a ferromagnetic insulator adjacent to a superconducting layer (S) (Sec. II). We discuss several experimental situations with the help of a theoretical framework (see Secs. II.A and III.B) based on the quasiclassical formalism, with which one can account for both thermodynamical and nonequilibrium properties of such hybrid structures. In order to account for effects beyond quasiclassics, as, for example, strong spin polarization, we combine the quasiclassical equations with effective boundary conditions.

Out-of-equilibrium superconductivity by itself leads to a decoupling between the charge and energy degrees of freedom of the electronic transport. In this Colloquium we show that the combination between superconductivity and magnetism requires, on the one hand, a description of additional nonequilibrium modes, spin and spin energy, and, on the other hand, to couple them all. This leads to novel and intriguing phenomena with direct impact on the latest research activities and proposed future technologies based on superconductors and spin-dependent fields (Eschrig, 2011, 2015; Linder and Robinson, 2015). By using the theoretical formalism presented one can predict and explain phenomena such as the spin injection and relaxation (Sec. IV) in superconductors with an intrinsic exchange field along with their consequences in the transport properties. We also discuss a number of striking thermoelectric effects in superconductors with a spinsplitting field (Sec. V).

The best scenario for the phenomena and applications discussed here, and, in particular, for the thermoelectric effects, is FI-S systems where the spin splitting can be achieved without the need of an applied magnetic field. Hence it becomes important to look for ideal FI-S material combinations. So far europium chalcogenides (EuO, EuS, and EuSe) together with aluminum films have shown large splittings and hence these are the best combination. In addition, thin films of $\mathrm{EuO}$ or EuS can be used as almost perfect spin filters (see Table I) and hence they are good candidates for realizing the near-optimal heat engines proposed in Sec. V. One of the main challenges from this perspective is to find FI-S combinations with large superconducting critical temperature and simultaneously a large spin splitting. Superconductors such as $\mathrm{Nb}$ or $\mathrm{Pb}$, on the one hand, increase $T_{C}$ with respect to Al-based structures, but, on the other hand, the spin-orbit coupling may spoil the sharp splitting as discussed in Sec. II. Recent experiments on GdN-NbN suggest large splittings (Pal and Blamire, 2015) but further research in this direction is needed.

In Sec. IV.D we discuss the dynamics of spin-split superconductors in rf fields. Historically, magnetic resonance effects in superconductors are well studied, but fewer experiments have probed spin-split thin films.

Besides the effects discussed in this Colloquium, several theoretical studies made striking predictions in mesoscopic systems with spin-split superconductors, such as the creation of highly polarized spin currents (Huertas-Hernando, Nazarov, and Belzig, 2002; Giazotto and Taddei, 2008; Giazotto and Bergeret, 2013b), large supercurrents in FI-S-I-S-FI junctions (Bergeret, Volkov, and Efetov, 2001a), junctions with switchable current-phase relations (Strambini, Bergeret, and Giazotto, 2015), and an almost ideal heat valve based on S-FI elements (Giazotto and Bergeret, 2013a).

Although many of the transport phenomena in spin-split superconductors are now well understood, we foresee a number of exciting avenues for future research. 
One further perspective of the present work is the extension of the Keldysh quasiclassical formalism to include magnetoelectric effects associated with the spin-orbit coupling (SOC). For a linear in momentum SOC the generalization of this can be done by introducing an effective $S U(2)$ gauge potential. The quasiclassical equations in this case were derived by Bergeret and Tokatly $(2013,2014,2016)$. Effects such as the spin Hall and spin-galvanic effect in superconductors have been studied in the equilibrium case (Konschelle, Tokatly, and Sebastián Bergeret, 2015). Extending these results to a nonequilibrium situation, and also to time-dependent fields, would be an interesting further development and would allow for a detailed study of the well-controlled nonlinearities associated with these effects in superconductors. The first steps in this direction have been taken by Espedal et al. (2017).

Recent discoveries of Skyrmionic states in chiral magnets (Nagaosa and Tokura, 2013) have attracted a lot of attention due to the effects resulting from the interplay of magnetism and SOC (Soumyanarayanan et al., 2016) which can induce chiral Dzyaloshinskii-Moriya interactions between magnetic moments. Currently it is interesting to study these effects in the presence of the additional component-superconductivity, when the exchange interaction is mediated by the Cooper pairs (de Gennes, 1966). One can expect that in such systems superconductivity can induce a nontrivial magnetic ordering and dynamics. These effects can show up in various systems including ferromagnet/superconductor bilayers, surface magnetic adatoms, and bulk magnetic impurities inducing the localized Yu-Shiba-Rusinov states modified by the SOC (Pershoguba et al., 2015).

Superconducting structures with strong spin-orbit coupling and exchange fields are also of much interest in view of engineering a platform for realization of topological phases and Majorana bound states (Hasan and Kane, 2010; Qi and Zhang, 2011; Alicea, 2012; Beenakker, 2013). Understanding and controlling the behavior and relaxation of nonequilibrium quasiparticles in these systems is also of importance, not least because of their influence on the prospects of solid-state topological quantum computation (Nayak et al., 2008).

This Colloquium focuses exclusively on the nonequilibrium properties of superconductors in proximity to magnets. We expect the inclusion of the magnetization dynamics and its coupling to the electronic degrees of freedom via the reciprocal effects of spin transfer torque and spin pumping (Tserkovnyak et al., 2005) in the far-from-equilibrium regime to lead to completely new type of physics, as the two types of order parameters affect each other. The coupling of the supercurrent on magnetization dynamics and texture has been studied during the past decade (Waintal and Brouwer, 2002; Houzet, 2008; Richard, Houzet, and Meyer, 2012), but the work where both systems are out of equilibrium has been mainly concentrated on Josephson junctions (Hikino et al., 2011; Holmqvist, Teber, and Fogelström, 2011; Mai et al., 2011; Holmqvist, Fogelström, and Belzig, 2014; Kulagina and Linder, 2014) and much less attention has been paid to quasiparticle effects (Skadsem et al., 2011; Linder et al., 2012; Trif and Tserkovnyak, 2013).

Besides the rich physics offered by spin-split superconductors, they have been long used as tools to characterize equilibrium properties of magnets, especially their spin polarization. In this Colloquium (see the end of Sec. V.B) we outlined two further possibilities related to their large thermoelectric response: accurate radiation sensing and noninvasive scanning thermometry. We believe there are also many other avenues to be uncovered, opened by the possibility for realizing a controlled combination of magnetism and superconductivity.

\section{ACKNOWLEDGMENTS}

We thank Faluke Aikebaier, Marco Aprili, Detlef Beckmann, Wolfgang Belzig, Alexander Bobkov, Irina Bobkova, Matthias Eschrig, Yuri Galperin, Francesco Giazotto, Vitaly Golovach, Kalle Kansanen, Alexander Mel'nikov, Jagadeesh Moodera, Risto Ojajärvi, Asier Ozaeta, Charis Quay, Jason Robinson, Mikel Rouco, and Elia Strambini for useful discussions. This work was supported by the Academy of Finland Center of Excellence (Project No. 284594), Research Fellow (Project No. 297439), and Key Funding (Project No. 305256) programs, the Spanish Ministerio de Economía y Competitividad (MINECO, Project No. FIS2014-55987-P), the Spanish Ministerio de Ciencia, Innovación y Universidades (MICINN, Project No. FIS201782804-P), and the European Research Council under the European Union's Seventh Framework Program (FP7/20072013)/ERC Grant agreements No. 240362-HEATTRONICS and No. 615187-COMANCHE.

\section{REFERENCES}

Aasen, David, et al., 2016, "Milestones toward Majorana-based quantum computing," Phys. Rev. X 6, 031016.

Abrikosov, A. A., and L.P. Gor'kov, 1960a, "Contribution to the theory of superconducting alloys with paramagnetic impurities," Zh. Eksp. Teor. Fiz. 39, 1781 [Sov. Phys. JETP 12, 1243 (1961)].

Abrikosov, A. A., and L. P. Gor'kov, 1960b, "On the problem of the Knight shift in superconductors," Zh. Eksp. Teor. Fiz. 39, 480 [Sov. Phys. JETP 12, 337 (1961), http://www.jetp.ac.ru/cgi-bin/e/index/ e/12/2/p337?a=list].

Abrikosov, A. A., and L. P. Gor'kov, 1962, "Spin-orbit interaction and the Knight shift in superconductors," Zh. Eksp. Teor. Fiz. 42, 1088 [Sov. Phys. JETP 15, 752 (1962), http://www.jetp.ac.ru/cgibin/e/index/e/15/4/p752?a=list].

Aikebaier, Faluke, Mihail A. Silaev, and T. T. Heikkilä, 2018, "Supercurrent induced charge-spin conversion in spin-split superconductors," Phys. Rev. B 98, 024516.

Alicea, J., 2012, "New directions in the pursuit of Majorana fermions in solid state systems," Rep. Prog. Phys. 75, 076501.

Anthore, A., H. Pothier, and D. Esteve, 2003, "Density of states in a superconductor carrying a supercurrent," Phys. Rev. Lett. 90, 127001.

Aoi, K., and J. C. Swihart, 1970, "Theory of electron spin resonance in type-I superconductors," Phys. Rev. B 2, 2555-2560.

Artemenko, S. N., and A.F. Volkov, 1979, "Electric fields and collective oscillations in superconductors," Sov. Phys. Usp. 22, 295.

Ashcroft, N.W., and N.D. Mermin, 1976, Solid State Physics (Saunders College, Philadelphia).

Baibich, M. N., J. M. Broto, A. Fert, F. Nguyen Van Dau, F. Petroff, P. Etienne, G. Creuzet, A. Friederich, and J. Chazelas, 1988, "Giant 
magnetoresistance of $(001) \mathrm{Fe} /(001) \mathrm{Cr}$ magnetic superlattices," Phys. Rev. Lett. 61, 2472-2475.

Bardeen, John, Leon N. Cooper, and John Robert Schrieffer, 1957, "Theory of superconductivity," Phys. Rev. 108, 1175.

Bathen, Marianne Etzelmüller, and Jacob Linder, 2017, "Spin Seebeck effect and thermoelectric phenomena in superconducting hybrids with magnetic textures or spin-orbit coupling," Sci. Rep. 7, 41409.

Beckmann, D., 2016, "Spin manipulation in nanoscale superconductors," J. Phys. Condens. Matter 28, 163001.

Beckmann, D., H. B. Weber, and H. v. Löhneysen, 2004, "Evidence for crossed Andreev reflection in superconductor-ferromagnet hybrid structures," Phys. Rev. Lett. 93, 197003.

Beenakker, C. W. J., 2013, "Search for Majorana fermions in superconductors," Annu. Rev. Condens. Matter Phys. 4, 113-136.

Belzig, Wolfgang, Frank K. Wilhelm, Christoph Bruder, Gerd Schön, and Andrei D. Zaikin, 1999, "Quasiclassical Green's function approach to mesoscopic superconductivity," Superlattices Microstruct. 25, 1251-1288.

Bergeret, F. S., K. B. Efetov, and A. I. Larkin, 2000, "Nonhomogeneous magnetic order in superconductor-ferromagnet multilayers," Phys. Rev. B 62, 11872-11878.

Bergeret, F. S., and F. Giazotto, 2014, "Manifestation of a spinsplitting field in a thermally biased Josephson junction," Phys. Rev. B 89, 054505.

Bergeret, F. S., and I. V. Tokatly, 2013, "Singlet-triplet conversion and the long-range proximity effect in superconductor-ferromagnet structures with generic spin dependent fields," Phys. Rev. Lett. 110, 117003.

Bergeret, F. S., and I. V. Tokatly, 2014, "Spin-orbit coupling as a source of long-range triplet proximity effect in superconductorferromagnet hybrid structures," Phys. Rev. B 89, 134517.

Bergeret, F. S., and I. V. Tokatly, 2016, "Manifestation of extrinsic spin Hall effect in superconducting structures: Nondissipative magnetoelectric effects," Phys. Rev. B 94, 180502.

Bergeret, F.S., A. Verso, and A.F. Volkov, 2012, "Electronic transport through ferromagnetic and superconducting junctions with spin-filter tunneling barriers," Phys. Rev. B 86, 214516.

Bergeret, F. S., A. F. Volkov, and K. B. Efetov, 2001a, "Enhancement of the Josephson current by an exchange field in superconductorferromagnet structures," Phys. Rev. Lett. 86, 3140.

Bergeret, F. S., A. F. Volkov, and K. B. Efetov, 2001b, "Long-range proximity effects in superconductor-ferromagnet structures," Phys. Rev. Lett. 86, 4096.

Bergeret, F. S., A. F. Volkov, and K. B. Efetov, 2004, "Induced ferromagnetism due to superconductivity in superconductorferromagnet structures," Phys. Rev. B 69, 174504.

Bergeret, F. S., Anatoly F. Volkov, and Konstantin B. Efetov, 2005, "Odd triplet superconductivity and related phenomena in superconductor-ferromagnet structures," Rev. Mod. Phys. 77, 13211373.

Bergeret, F. Sebastian, Mikhail Silaev, Pauli Virtanen, and Tero T. Heikkila, 2017, "Nonequilibrium effects in superconductors with a spin-splitting field," arXiv:1706.08245.

Binasch, G., P. Grünberg, F. Saurenbach, and W. Zinn, 1989, "Enhanced magnetoresistance in layered magnetic structures with antiferromagnetic interlayer exchange," Phys. Rev. B 39, 48284830.

Bobkova, I. V., and A. M. Bobkov, 2015, "Long-range spin imbalance in mesoscopic superconductors under Zeeman splitting," JETP Lett. 101, 118.
Bobkova, I. V., and A. M. Bobkov, 2016, "Injection of nonequilibrium quasiparticles into Zeeman-split superconductors: a way to create long-range spin imbalance,” Phys. Rev. B 93, 024513.

Bobkova, I. V., and A. M. Bobkov, 2017, "Thermospin effects in superconducting heterostructures,” Phys. Rev. B 96, 104515.

Brataas, A., G. Bauer, and P. Kelly, 2006, "Non-collinear magnetoelectronics," Phys. Rep. 427, 157-255.

Bruno, Ronald, C., and Brian B. Schwartz, 1973, "Magnetic field splitting of the density of states of thin superconductors," Phys. Rev. B 8, 3161-3178.

Buzdin, A. I., 2005, "Proximity effects in superconductor-ferromagnet heterostructures," Rev. Mod. Phys. 77, 935-976.

Buzdin, A. I., and L. N. Bulaevskii, 1988, "Ferromagnetic film on the surface of a superconductor: Possible onset of inhomogeneous magnetic ordering," Sov. Phys. JETP 67, 576-578 [http://www.jetp .ac.ru/cgi-bin/e/index/e/67/3/p576?a=list].

Buzdin, A. I., L. N. Bulaevskii, and S. V. Panyukov, 1982, "Criticalcurrent oscillations as a function of the exchange field and thickness of the ferromagnetic metal (f) in an sfs josephson junction," JETP Lett. 35, 178 [http://www.jetpletters.ac.ru/ps/1314/ article_19853.pdf].

Cadden-Zimansky, P., Z. Jiang, and V. Chandrasekhar, 2007, "Charge imbalance, crossed Andreev reflection and elastic cotunnelling in ferromagnet/superconductor/normal-metal structures," New J. Phys. 9, 116.

Carlson, R. V., and A. M. Goldman, 1973, "Superconducting orderparameter fluctuations below $T_{c}$," Phys. Rev. Lett. 31, 880.

Chandrasekhar, B. S., 1962, "A note on the maximum critical field of high-field superconductors," Appl. Phys. Lett. 1, 7-8.

Chtchelkatchev, N., and I. Burmistrov, 2008, "Energy relaxation in the spin-polarized disordered electron liquid," Phys. Rev. Lett. 100, 206804.

Clarke, John, 1972, "Experimental observation of pair-quasiparticle potential difference in nonequilibrium superconductors," Phys. Rev. Lett. 28, 1363-1366.

Clogston, A. M., 1962, "Upper limit for the critical field in hard superconductors," Phys. Rev. Lett. 9, 266-267.

Cottet, Audrey, Daniel Huertas-Hernando, Wolfgang Belzig, and Yu. V. Nazarov, 2009, "Spin-dependent boundary conditions for isotropic superconducting Green functions," Phys. Rev. B 80, 184511.

de Gennes, P. G., 1966, "Coupling between ferromagnets through a superconducting layer," Phys. Lett. 23, 10-11.

de Gennes, P. G., 1999, Superconductivity of Metals and Alloys, Advanced book classics (Perseus, Cambridge, MA).

Dejene, F. K., J. Flipse, G. E. W. Bauer, and B. J. van Wees, 2013, "Spin heat accumulation and spin-dependent temperatures in nanopillar spin valves," Nat. Phys. 9, 636.

Demler, E. A., G. B. Arnold, and M. R. Beasley, 1997, "Superconducting proximity effects in magnetic metals," Phys. Rev. B 55, 15174.

Dimitrova, O. V., and V. E. Kravtsov, 2008, "Infrared catastrophe in a two-quasiparticle collision integral," JETP Lett. 86, 670-676.

Dynes, R. C., J. P. Garno, G. B. Hertel, and T. P. Orlando, 1984, "Tunneling study of superconductivity near the metal-insulator transition," Phys. Rev. Lett. 53, 2437-2440.

Eilenberger, Gert, 1968, “Transformation of Gorkov's equation for type II superconductors into transport-like equations," Z. Phys. A 214, 195-213.

Eliashberg, G. M., 1970, "Film superconductivity stimulated by a high-frequency field," JETP Lett. 11, 114 [http://www.jetpletters.ac .ru/ps/1716/article_26086.pdf]. 
Eliashberg, G. M., 1972, "Inelastic electron collisions and nonequilibrium stationary states in superconductors," Sov. Phys. JETP 34, 668 [http://www.jetp.ac.ru/cgi-bin/dn/e_034_03_0668.pdf].

Eltschka, Matthias, Berthold Jäck, Maximilian Assig, Oleg V. Kondrashov, Mikhail A. Skvortsov, Markus Etzkorn, Christian R. Ast, and Klaus Kern, 2014, "Probing absolute spin polarization at the nanoscale," Nano Lett. 14, 7171-7174.

Eltschka, Matthias, Berthold Jäck, Maximilian Assig, Oleg V. Kondrashov, Mikhail A. Skvortsov, Markus Etzkorn, Christian R. Ast, and Klaus Kern, 2015, "Superconducting scanning tunneling microscopy tips in a magnetic field: Geometry-controlled order of the phase transition," Appl. Phys. Lett. 107, 122601.

Eschrig, Matthias, 2011, "Spin-polarized supercurrents for spintronics," Phys. Today 64, No. 1, 43.

Eschrig, Matthias, 2015, "Spin-polarized supercurrents for spintronics: a review of current progress," Rep. Prog. Phys. 78, 104501.

Eschrig, Matthias, Audrey Cottet, Wolfgang Belzig, and Jacob Linder, 2015, "General boundary conditions for quasiclassical theory of superconductivity in the diffusive limit: application to strongly spin-polarized systems," New J. Phys. 17, 083037.

Espedal, Camilla, Peter Lange, Severin Sadjina, A. G. Mal'shukov, and Arne Brataas, 2017, "Spin hall effect and spin swapping in diffusive superconductors," Phys. Rev. B 95, 054509.

Feigel'man, M. V., A. I. Larkin, and M. A. Skvortsov, 2000, "Keldysh action for disordered superconductors," Phys. Rev. B 61, 12361-12388.

Fulde, P., and R. A. Ferrell, 1964, "Superconductivity in a strong spin-exchange field," Phys. Rev. 135, A550-A563.

Galperin, Yu. M., V. L. Gurevich, and V. I. Kozub, 1974, "Thermoelectric effects in superconductors," Sov. Phys. JETP 39, 680 [http://www.jetp.ac.ru/cgi-bin/dn/e_039_04_0680.pdf].

Giaever, I., and K. Megerle, 1961, "Study of superconductors by electron tunneling," Phys. Rev. 122, 1101-1111.

Giazotto, F., and F. S. Bergeret, 2013a, "Phase-tunable colossal magnetothermal resistance in ferromagnetic Josephson valves," Appl. Phys. Lett. 102, 132603-132605.

Giazotto, F., and F. S. Bergeret, 2013b, "Quantum interference hybrid spin-current injector,” Appl. Phys. Lett. 102, 162406.

Giazotto, F., T. T. Heikkilä, and F. S. Bergeret, 2015, "Very large thermophase in ferromagnetic Josephson junctions," Phys. Rev. Lett. 114, 067001.

Giazotto, F., P. Solinas, A. Braggio, and F. S. Bergeret, 2015, "Ferromagnetic-insulator-based superconducting junctions as sensitive electron thermometers," Phys. Rev. Applied 4, 044016.

Giazotto, F., and F. Taddei, 2008, "Superconductors as spin sources for spintronics," Phys. Rev. B 77, 132501.

Giazotto, Francesco, Tero T. Heikkilä, Arttu Luukanen, Alexander M. Savin, and Jukka P. Pekola, 2006, "Opportunities for mesoscopics in thermometry and refrigeration: Physics and applications," Rev. Mod. Phys. 78, 217-274.

Ginzburg, V. L., 1944, "On the thermoelectric phenomena in superconductors," Zh. Eksp. Teor. Fiz. 14, 177.[J. Phys. USSR 8, 148 (1944)].

Ginzburg, V. L., 1957, "Ferromagnetic superconductors," Sov. Phys. JETP 4, 153 [http://www.jetp.ac.ru/cgi-bin/dn/e_004_02_0153 .pdf].

Grimaldi, Claudio, and Peter Fulde, 1997, "Nonequilibrium superconductivity in spin-polarized superconducting tunneling junctions," Phys. Rev. B 56, 2751-2763.

Gu, J. Y., J. A. Caballero, R. D. Slater, R. Loloee, and W. P. Pratt, Jr., 2002, "Direct measurement of quasiparticle evanescent waves in a dirty superconductor," Phys. Rev. B 66, 140507.
Hao, X., J. S. Moodera, and R. Meservey, 1990, "Spin-filter effect of ferromagnetic europium sulfide tunnel barriers," Phys. Rev. B 42, 8235-8243.

Hasan, M.Z., and C.L. Kane, 2010, "Colloquium: Topological insulators," Rev. Mod. Phys. 82, 3045-3067.

Heikkilä, T. T., R. Ojajärvi, I. Maasilta, F. Giazotto, and F. S. Bergeret, 2017, "Thermoelectric radiation detector based on superconductor/ferromagnet systems," Phys. Rev. Applied (in press), arXiv: 1709.08856

Higgs, Peter W., 1964, "Broken symmetries and the masses of gauge bosons," Phys. Rev. Lett. 13, 508-509.

Hikino, Shin-ichi, Michiyasu Mori, Saburo Takahashi, and Sadamichi Maekawa, 2011, "Composite excitation of Josephson phase and spin waves in Josephson junctions with ferromagnetic insulator,” J. Phys. Soc. Jpn. 80, 074707.

Hoffman, Silas, Koji Sato, and Yaroslav Tserkovnyak, 2013, "Landau-Lifshitz theory of the longitudinal spin Seebeck effect," Phys. Rev. B 88, 064408.

Holmqvist, C., M. Fogelström, and W. Belzig, 2014, "Spin-polarized Shapiro steps and spin-precession-assisted multiple Andreev reflection,” Phys. Rev. B 90, 014516.

Holmqvist, C., S. Teber, and M. Fogelström, 2011, "Nonequilibrium effects in a Josephson junction coupled to a precessing spin," Phys. Rev. B 83, 104521.

Houzet, Manuel, 2008, "Ferromagnetic Josephson junction with precessing magnetization,” Phys. Rev. Lett. 101, 057009.

Hübler, F., J. C. Lemyre, D. Beckmann, and H. v. Löhneysen, 2010, "Charge imbalance in superconductors in the low-temperature limit," Phys. Rev. B 81, 184524.

Hübler, F., M. J. Wolf, D. Beckmann, and H. v. Löhneysen, 2012, "Long-range spin-polarized quasiparticle transport in mesoscopic Al superconductors with a Zeeman splitting," Phys. Rev. Lett. 109, 207001.

Huertas-Hernando, Daniel, Yu. V. Nazarov, and W. Belzig, 2002, "Absolute spin-valve effect with superconducting proximity structures," Phys. Rev. Lett. 88, 047003.

Hwang, Sun-Yong, Rosa López, and David Sánchez, 2016, "Large thermoelectric power and figure of merit in a ferromagnetic-quantum dot-superconducting device," Phys. Rev. B 94, 054506.

Ivlev, B. I., S. G. Lisitsyn, and G. M. Eliashberg, 1973, "Nonequilibrium excitations in superconductors in high-frequency fields," J. Low Temp. Phys. 10, 449-468.

Izyumov, Yu. A., Yu. N. Proshin, and M. G. Khusainov, 2002, "Competition between superconductivity and magnetism in ferromagnet/superconductor heterostructures," Phys. Usp. 45, 109-148.

Jacquod, Philippe, Robert S. Whitney, Jonathan Meair, and Markus Büttiker, 2012, "Onsager relations in coupled electric, thermoelectric, and spin transport: The tenfold way," Phys. Rev. B 86, 155118. Jedema, F. J., A. T. Filip, and B. J. van Wees, 2001, “Electrical spin injection and accumulation at room temperature in an all-metal mesoscopic spin valve," Nature (London) 410, 345.

Jedema, F. J., H. B. Heersche, A. T. Filip, J. J. A. Baselmans, and B. J. van Wees, 2002, "Electrical detection of spin precession in a metallic mesoscopic spin valve," Nature (London) 416, 713.

Jedema, F. J., M. S. Nijboer, A. T. Filip, and B. J. Van Wees, 2003, "Spin injection and spin accumulation in all-metal mesoscopic spin valves," Phys. Rev. B 67, 085319.

Jiang, J. S., Dragomir Davidović, Daniel H. Reich, and C. L. Chien, 1996, "Superconducting transition in nb/gd/nb trilayers," Phys. Rev. B 54, 6119. 
Johnson, Mark, 1994, "Spin coupled resistance observed in ferromagnet-superconductor-ferromagnet trilayers," Appl. Phys. Lett. 65, 1460-1462.

Johnson, Mark, and R. H. Silsbee, 1985, "Interfacial charge-spin coupling: Injection and detection of spin magnetization in metals," Phys. Rev. Lett. 55, 1790-1793.

Kalenkov, M. S., A. D. Zaikin, and L. S. Kuzmin, 2012, "Theory of a large thermoelectric effect in superconductors doped with magnetic impurities," Phys. Rev. Lett. 109, 147004.

Kamenev, A., and A. Levchenko, 2009, "Keldysh technique and nonlinear $\sigma$-model: basic principles and applications," Adv. Phys. 58, 197.

Kaplan, S. B., C. C. Chi, D. N. Langenberg, J. J. Chang, S. Jafarey, and D. J. Scalapino, 1976, "Quasiparticle and phonon lifetimes in superconductors," Phys. Rev. B 14, 4854-4873.

Khusainov, M. G., 1996, "Indirect RKKY exchange and magnetic states of ferromagnet-superconductor superlattices," Zh. Eksp. Teor. Fiz. 109, 524 [JETP 82, 278 (1996), http://www.jetp.ac.ru/ cgi-bin/dn/e_082_02_0278.pdf].

Kim, Sang Il., et al., 2015, "Dense dislocation arrays embedded in grain boundaries for high-performance bulk thermoelectrics," Science 348, 109-114.

Kivelson, S. A., and D.S. Rokhsar, 1990, "Bogoliubov quasiparticles, spinons, and spin-charge decoupling in superconductors," Phys. Rev. B 41, 11693-11696.

Klapwijk, T. M., J. N. Van den Bergh, and J.E. Mooij, 1977, "Radiation-stimulated superconductivity," J. Low Temp. Phys. 26, 385-405.

Kolenda, S., C. Sürgers, G. Fischer, and D. Beckmann, 2017, "Thermoelectric effects in superconductor-ferromagnet tunnel junctions on europium sulfide," Phys. Rev. B 95, 224505.

Kolenda, S., M. J. Wolf, and D. Beckmann, 2016, "Observation of thermoelectric currents in high-field superconductor-ferromagnet tunnel junctions," Phys. Rev. Lett. 116, 097001.

Konschelle, François, Ilya V. Tokatly, and F. Sebastián Bergeret, 2015, "Theory of the spin-galvanic effect and the anomalous phase shift $\varphi_{0}$ in superconductors and Josephson junctions with intrinsic spin-orbit coupling," Phys. Rev. B 92, 125443.

Kontos, T., M. Aprili, J. Lesueur, and X. Grison, 2001, "Inhomogeneous superconductivity induced in a ferromagnet by proximity effect," Phys. Rev. Lett. 86, 304.

Kopnin, N. B., 2001, Theory of nonequilibrium superconductivity, International series of monographs on physics No. 110 (Oxford University Press, New York).

Krishtop, Tatiana, Manuel Houzet, and Julia S. Meyer, 2015, "Nonequilibrium spin transport in Zeeman-split superconductors," Phys. Rev. B 91, 121407.

Kulagina, Iryna, and Jacob Linder, 2014, "Spin supercurrent, magnetization dynamics, and $\varphi$-state in spin-textured Josephson junctions," Phys. Rev. B 90, 054504.

Kuprianov, M. Yu, and V. F. Lukichev, 1988, "Influence of boundary transparency on the critical current of "dirty" SS'S structures," Sov. Phys. JETP 67, 1163-1168 [http://www.jetp.ac.ru/cgi-bin/dn/ e_067_06_1163.pdf].

Lambert, C. J., R. Raimondi, V. Sweeney, and A. F. Volkov, 1997, "Boundary conditions for quasiclassical equations in the theory of superconductivity," Phys. Rev. B 55, 6015-6021.

Langenberg, D. N., and A. I. Larkin, 1986, Nonequilibrium superconductivity (North-Holland, Amsterdam).

Larkin, A. I., and Yuri N. Ovchinnikov, 1965, "Inhomogeneous state of superconductors," Sov. Phys. JETP 20, 762-769.
Leivo, M. M., J. P. Pekola, and D. V. Averin, 1996, "Efficient Peltier refrigeration by a pair of normal metal/insulator/superconductor junctions," Appl. Phys. Lett. 68, 1996-1998.

Linder, Jacob, and Marianne Etzelmüller Bathen, 2016, "Spin caloritronics with superconductors: Enhanced thermoelectric effects, generalized Onsager response-matrix, and thermal spin currents," Phys. Rev. B 93, 224509.

Linder, Jacob, Arne Brataas, Zahra Shomali, and Malek Zareyan, 2012, "Spin-transfer and exchange torques in ferromagnetic superconductors," Phys. Rev. Lett. 109, 237206.

Linder, Jacob, and Jason W. A. Robinson, 2015, "Superconducting spintronics," Nat. Phys. 11, 307-315.

Machon, P., M. Eschrig, and W. Belzig, 2013, "Nonlocal thermoelectric effects and nonlocal Onsager relations in a three-terminal proximity-coupled superconductor-ferromagnet device," Phys. Rev. Lett. 110, 047002.

Machon, P., M. Eschrig, and W. Belzig, 2014, "Giant thermoelectric effects in a proximity-coupled superconductor-ferromagnet device," New J. Phys. 16, 073002.

Mai, S., E. Kandelaki, A. F. Volkov, and K. B. Efetov, 2011, "Interaction of Josephson and magnetic oscillations in Josephson tunnel junctions with a ferromagnetic layer," Phys. Rev. B 84, 144519.

Maki, K., 1966, "Effect of Pauli paramagnetism on magnetic properties of high-field superconductors," Phys. Rev. 148, 362.

Maki, K., 1973, "Theory of electron-spin resonance in gapless superconductors," Phys. Rev. B 8, 191-199.

Marchegiani, G., P. Virtanen, F. Giazotto, and M. Campisi, 2016, "Self-oscillating Josephson quantum heat engine," Phys. Rev. Applied 6, 054014.

Meissner, W.Z., 1927, "Das elektrische Verhalten der Metalle im Temperaturgebiet des flüssigen Heliums," Z. ges. Kälte-Industrie 34, 197.

Menges, Fabian, Philipp Mensch, Heinz Schmid, Heike Riel, Andreas Stemmer, and Bernd Gotsmann, 2016, "Temperature mapping of operating nanoscale devices by scanning probe thermometry," Nat. Commun. 7, 10874.

Meservey, R., D. Paraskevopoulos, and P. M. Tedrow, 1980, "Tunneling measurements of conduction-electron-spin polarization in heavy rare-earth metals," Phys. Rev. B 22, 1331-1337.

Meservey, R., P. M. Tedrow, and Ronald C. Bruno, 1975, "Tunneling measurements on spin-paired superconductors with spin-orbit scattering," Phys. Rev. B 11, 4224.

Meservey, R., P. M. Tedrow, and Peter Fulde, 1970, "Magnetic field splitting of the quasiparticle states in superconducting aluminum films," Phys. Rev. Lett. 25, 1270-1272.

Meservey, Robert, and P. M. Tedrow, 1994, "Spin-polarized electron tunneling," Phys. Rep. 238, 173-243.

Moodera, J.S., X. Hao, G. A. Gibson, and R. Meservey, 1988, "Electron-spin polarization in tunnel junctions in zero applied field with ferromagnetic EuS barriers," Phys. Rev. Lett. 61, 637-640.

Moodera, J. S., R. Meservey, and X. Hao, 1993, "Variation of the electron-spin polarization in EuSe tunnel junctions from zero to near $100 \%$ in a magnetic field," Phys. Rev. Lett. 70, 853-856.

Moodera, Jagadeesh S., Tiffany S. Santos, and Taro Nagahama, 2007, "The phenomena of spin-filter tunnelling," J. Phys. Condens. Matter 19, 165202.

Moodera, Jagadeesh Subbaiah, Lisa R. Kinder, Terrilyn M. Wong, and R. Meservey, 1995, "Large magnetoresistance at room temperature in ferromagnetic thin film tunnel junctions," Phys. Rev. Lett. 74, 3273 . 
Morten, J. P., A. Brataas, and W. Belzig, 2004, "Spin transport in diffusive superconductors," Phys. Rev. B 70, 212508.

Morten, Jan Petter, Arne Brataas, and Wolfgang Belzig, 2005, "Spin transport and magnetoresistance in ferromagnet/superconductor/ ferromagnet spin valves," Phys. Rev. B 72, 014510.

Münzenberg, M., and J.S. Moodera, 2004, "Superconductorferromagnet tunneling measurements indicates p-spin and d-spin currents," Phys. Rev. B 70, 060402.

Nagaosa, N., and Y. Tokura, 2013, "Topological properties and dynamics of magnetic skyrmions," Nat. Nanotechnol. 8, 899-911.

Nahum, M., T. M. Eiles, and John M. Martinis, 1994, "Electronic microrefrigerator based on a normal-insulator-superconductor tunnel junction," Appl. Phys. Lett. 65, 3123-3125.

Narozhny, B. N., I. L. Aleiner, and B. L. Altshuler, 1999, “Theory of interaction effects in normal-metal-superconductor junctions out of equilibrium," Phys. Rev. B 60, 7213-7227.

Nayak, Chetan, Steven H. Simon, Ady Stern, Michael Freedman, and Sankar Das Sarma, 2008, "Non-Abelian anyons and topological quantum computation," Rev. Mod. Phys. 80, 1083-1159.

Nazarov, Yu. V., 1999, "Novel circuit theory of Andreev reflection," Superlattices Microstruct. 25, 1221-1231.

Nemes, N. M., J. E. Fischer, G. Baumgartner, L. Forró, T. Fehér, G. Oszlányi, F. Simon, and A. Jánossy, 2000, "Conduction-electron spin resonance in the superconductor $\mathrm{k}_{3} \mathrm{c}_{60}$," Phys. Rev. B 61, 7118-7121.

Nielsen, J. Beyer, C. J. Pethick, J. Rammer, and H. Smith, 1982, "Pair breaking and charge relaxation in superconductors," J. Low Temp. Phys. 46, 565-597.

Onsager, Lars, 1931, "Reciprocal relations in irreversible processes. I.," Phys. Rev. 37, 405-426.

Ozaeta, A., P. Virtanen, F. S. Bergeret, and T. T. Heikkilä, 2014, "Predicted very large thermoelectric effect in ferromagnet-superconductor junctions in the presence of a spin-splitting magnetic field," Phys. Rev. Lett. 112, 057001.

Pal, Avradeep, and M. G. Blamire, 2015, "Large interfacial exchange fields in a thick superconducting film coupled to a spin-filter tunnel barrier," Phys. Rev. B 92, 180510.

Paraskevopoulos, D., R. Meservey, and P. M. Tedrow, 1977, "Spin polarization of electrons tunneling from $3 d$ ferromagnetic metals and alloys," Phys. Rev. B 16, 4907-4919.

Pekola, J. P., T. T. Heikkilä, A. M. Savin, J. T. Flyktman, F. Giazotto, and F. W. J. Hekking, 2004, "Limitations in cooling electrons using normal-metal-superconductor tunnel junctions," Phys. Rev. Lett. 92, 056804

Pershoguba, S. S., K. Björnson, A. M. Black-Schaffer, and A. V. Balatsky, 2015, "Currents induced by magnetic impurities in superconductors with spin-orbit coupling," Phys. Rev. Lett. 115, 116602.

Poli, Ninos, Jan Petter Morten, Mattias Urech, Arne Brataas, David B. Haviland, and Vladislav Korenivski, 2008, "Spin injection and relaxation in a mesoscopic superconductor,' Phys. Rev. Lett. 100, 136601.

Qi, Xiao-Liang, and Shou-Cheng Zhang, 2011, "Topological insulators and superconductors," Rev. Mod. Phys. 83, 1057-1110.

Quay, C.H. L., and M. Aprili, 2018, "Out-of-equilibrium spin transport in mesoscopic superconductors," Phil. Trans. R. Soc. A 376, 20150342.

Quay, C. H. L., D. Chevallier, C. Bena, and M. Aprili, 2013, "Spin imbalance and spin-charge separation in a mesoscopic superconductor," Nat. Phys. 9, 84-88.

Quay, C. H. L., Y. Chiffaudel, C. Strunk, and M. Aprili, 2015, "Quasiparticle spin resonance and coherence in superconducting aluminium," Nat. Commun. 6, 8660.
Quay, C. H. L., C. Dutreix, D. Chevallier, C. Bena, and M. Aprili, 2016, "Frequency-domain measurement of the spin-imbalance lifetime in superconductors," Phys. Rev. B 93, 220501.

Rezaei, Ali, Akashdeep Kamra, Peter Machon, and Wolfgang Belzig, 2018, "Spin-flip enhanced thermoelectricity in superconductorferromagnet bilayers," New J. Phys. 20, 073034.

Richard, Caroline, Manuel Houzet, and Julia S. Meyer, 2012, "Andreev current induced by ferromagnetic resonance," Phys. Rev. Lett. 109, 057002.

Rouco, Mikel, Tero T. Heikkilä, and F. Sebastian Bergeret, 2018, "Electron refrigeration in hybrid structures with spin-split superconductors," Phys. Rev. B 97, 014529.

Ryazanov, V. V., V. A. Oboznov, A. Yu. Rusanov, A. V. Veretennikov, A. A. Golubov, and J. Aarts, 2001, "Coupling of two superconductors through a ferromagnet: evidence for a $\pi$ junction," Phys. Rev. Lett. 86, 2427.

Saint-James, D., G. Sarma, and E. J. Thomas, 1969, Type II Superconductivity, Commonwealth and International Library. Liberal Studies Divi (Elsevier, Science \& Technology, New York).

Schmid, Albert, and Gerd Schön, 1975, "Linearized kinetic equations and relaxation processes of a superconductor near $T_{c}$," J. Low Temp. Phys. 20, 207-227.

Senapati, Kartik, Mark G. Blamire, and Zoe H. Barber, 2011, "Spinfilter Josephson junctions," Nat. Mater. 10, 849-852.

Serene, J. W., and D. Rainer, 1983, "The quasiclassical approach to superfluid 3He," Phys. Rep. 101, 221.

Shelly, Connor D., Ekaterina A. Matrozova, and Victor T. Petrashov, 2016, "Resolving thermoelectric "paradox" in superconductors," Sci. Adv. 2, e1501250.

Shin, Y.-S., H.-J. Lee, and H.-W. Lee, 2005, "Spin relaxation in mesoscopic superconducting Al wires," Phys. Rev. B 71, 144513.

Silaev, Mihail, P. Virtanen, F. S. Bergeret, and T. T. Heikkilä, 2015, "Long-range spin accumulation from heat injection in mesoscopic superconductors with Zeeman splitting," Phys. Rev. Lett. 114, 167002.

Silaev, Mihail, P. Virtanen, T. T. Heikkilä, and F. S. Bergeret, 2015, "Spin Hanle effect in mesoscopic superconductors," Phys. Rev. B 91, 024506.

Singh, A., S. Voltan, K. Lahabi, and J. Aarts, 2015, "Colossal proximity effect in a superconducting triplet spin valve based on the half-metallic ferromagnet $\mathrm{cro}_{2}$," Phys. Rev. X 5, 021019.

Singh, Amrita, Charlotte Jansen, Kaveh Lahabi, and Jan Aarts, 2016, "High-quality $\mathrm{CrO}_{2}$ nanowires for dissipation-less spintronics," Phys. Rev. X 6, 041012.

Skadsem, Hans Joakim, Arne Brataas, Jan Martinek, and Yaroslav Tserkovnyak, 2011, "Ferromagnetic resonance and voltage-induced transport in normal metal-ferromagnet-superconductor trilayers," Phys. Rev. B 84, 104420.

Snyder, G. Jeffrey, and Tristan S. Ursell, 2003, "Thermoelectric efficiency and compatibility," Phys. Rev. Lett. 91, 148301.

Soumyanarayanan, A., N. Reyren, A. Fert, and C. Panagopoulos, 2016, "Emergent phenomena induced by spin-orbit coupling at surfaces and interfaces," Nature (London) 539, 509-517.

Strambini, E., F. S. Bergeret, and F. Giazotto, 2015, "Mesoscopic Josephson junctions with switchable current-phase relation," Europhys. Lett. 112, 17013.

Strambini, E., V. N. Golovach, G. De Simoni, J. S. Moodera, F. S. Bergeret, and F. Giazotto, 2017, "Revealing the magnetic proximity effect in eus/al bilayers through superconducting tunneling spectroscopy," Phys. Rev. Mater. 1, 054402.

Takahashi, S., and S. Maekawa, 2003, "Spin injection and detection in magnetic nanostructures," Phys. Rev. B 67, 052409. 
Tedrow, P. M., and R. Meservey, 1971, "Spin-dependent tunneling into ferromagnetic nickel," Phys. Rev. Lett. 26, 192-195.

Tedrow, P. M., and R. Meservey, 1973, "Spin polarization of electrons tunneling from films of $\mathrm{Fe}, \mathrm{Co}, \mathrm{Ni}$, and $\mathrm{Gd}$," Phys. Rev. B 7, 318.

Tedrow, P. M., J. E. Tkaczyk, and A. Kumar, 1986, "Spin-polarized electron tunneling study of an artificially layered superconductor with internal magnetic field: EuO-Al," Phys. Rev. Lett. 56, 17461749.

Tinkham, M., 1972, “Tunneling generation, relaxation, and tunneling detection of hole-electron imbalance in superconductors," Phys. Rev. B 6, 1747-1756.

Tinkham, M., and John Clarke, 1972, "Theory of pair-quasiparticle potential difference in nonequilibrium superconductors," Phys. Rev. Lett. 28, 1366-1369.

Tinkham, M., 1996, Introduction to superconductivity (McGrawHill, New York).

Tokuyasu, T., J. A. Sauls, and D. Rainer, 1988, "Proximity effect of a ferromagnetic insulator in contact with a superconductor," Phys. Rev. B 38, 8823-8833.

Torrey, H. C., 1956, "Bloch equations with diffusion terms," Phys. Rev. 104, 563-565.

Trif, Mircea, and Yaroslav Tserkovnyak, 2013, "Dynamic magnetoelectric effect in ferromagnet/superconductor tunnel junctions," Phys. Rev. Lett. 111, 087602.

Tserkovnyak, Yaroslav, Arne Brataas, Gerrit E.W. Bauer, and Bertrand I. Halperin, 2005, "Nonlocal magnetization dynamics in ferromagnetic heterostructures," Rev. Mod. Phys. 77, 13751421.

Uchida, K., M. Ishida, T. Kikkawa, A. Kirihara, T. Murakami, and E. Saitoh, 2014, "Longitudinal spin Seebeck effect: from fundamentals to applications," J. Phys. Condens. Matter 26, 343202 .

Usadel, Klaus D., 1970, “Generalized diffusion equation for superconducting alloys,” Phys. Rev. Lett. 25, 507-509.

van Bentum, P. J. M., and P. Wyder, 1986, "Far-infrared absorption of thin superconducting aluminum films in the pair-breaking and paramagnetic limits," Phys. Rev. B 34, 1582-1594.

Van Harlingen, D. J., D. F. Heidel, and J. C. Garland, 1980, "Experimental study of thermoelectricity in superconducting indium," Phys. Rev. B 21, 1842-1857.

van Son, P. C., H. van Kempen, and P. Wyder, 1987, "Boundary resistance of the ferromagnetic-nonferromagnetic metal interface," Phys. Rev. Lett. 58, 2271-2273.

Vier, D. C., and S. Schultz, 1983, "Observation of conduction electron spin resonance in both the normal and superconducting states of niobium," Phys. Lett. A 98, 283.

Villamor, Estitxu, Miren Isasa, Saül Vélez, Amilcar Bedoya-Pinto, Paolo Vavassori, Luis E. Hueso, F. Sebastián Bergeret, and Fèlix Casanova, 2015, "Modulation of pure spin currents with a ferromagnetic insulator," Phys. Rev. B 91, 020403.
Virtanen, P., T. T. Heikkilä, and F. S. Bergeret, 2016, "Stimulated quasiparticles in spin-split superconductors," Phys. Rev. B 93, 014512.

Waintal, Xavier, and Piet W. Brouwer, 2002, "Magnetic exchange interaction induced by a Josephson current," Phys. Rev. B 65, 054407.

Wakamura, T., N. Hasegawa, K. Ohnishi, Y. Niimi, and YoshiChika Otani, 2014, "Spin injection into a superconductor with strong spin-orbit coupling," Phys. Rev. Lett. 112, 036602.

Wellstood, F. C., C. Urbina, and John Clarke, 1994, "Hot-electron effects in metals," Phys. Rev. B 49, 5942-5955.

Wolf, M. J., F. Hübler, S. Kolenda, and D. Beckmann, 2014, "Charge and spin transport in mesoscopic superconductors," Beilstein J. Nanotechnol. 5, 180-185.

Wolf, M. J., F. Hübler, S. Kolenda, H. v. Löhneysen, and D. Beckmann, 2013, "Spin injection from a normal metal into a mesoscopic superconductor," Phys. Rev. B 87, 024517.

Wolf, M. J., C. Sürgers, G. Fischer, and D. Beckmann, 2014, "Spinpolarized quasiparticle transport in exchange-split superconducting aluminum on europium sulfide," Phys. Rev. B 90, 144509.

Xiong, Y. M., S. Stadler, P. W. Adams, and G. Catelani, 2011, "Spinresolved tunneling studies of the exchange field in EuS/Al bilayers," Phys. Rev. Lett. 106, 247001.

Yafet, Y., D. C. Vier, and S. Schultz, 1984, "Conduction electron spin resonance and relaxation in the superconducting state," J. Appl. Phys. 55, 2022-2024.

Yagi, Ryuta, 2006, "Charge imbalance observed in voltage-biased superconductor-normal tunnel junctions," Phys. Rev. B 73, 134507.

Yang, Hyunsoo, See-Hun Yang, Saburo Takahashi, Sadamichi Maekawa, and Stuart S. P. Parkin, 2010, "Extremely long quasiparticle spin lifetimes in superconducting aluminium using $\mathrm{MgO}$ tunnel spin injectors," Nat. Mater. 9, 586-593.

Yang, Tao, Takashi Kimura, and Yoshichika Otani, 2008, "Giant spin-accumulation signal and pure spin-current-induced reversible magnetization switching," Nat. Phys. 4, 851-854.

Yosida, Kei, 1958, "Paramagnetic susceptibility in superconductors," Phys. Rev. 110, 769.

Zaitsev, A. V., 1984, "Quasiclassical equations of the theory of superconductivity for contiguous metals and the properties of constricted microcontacts," Sov. Phys. JETP 59, 1015 [http:// www.jetp.ac.ru/cgi-bin/dn/e_059_05_1015.pdf].

Zhao, Erhai, Tomas Löfwander, and J. A. Sauls, 2004, "Nonequilibrium superconductivity near spin-active interfaces," Phys. Rev. B 70, 134510.

Zhao, Hui Lin, and Selman Hershfield, 1995, “Tunneling, relaxation of spin-polarized quasiparticles, and spin-charge separation in superconductors," Phys. Rev. B 52, 3632-3638.

Zhao, Li-Dong, et al., 2016, "Ultrahigh power factor and thermoelectric performance in hole-doped single-crystal SnSe," Science 351, 141-144. 